\title{
Rotation in Biphenyls with a Single ortho-Substituent
}

\author{
Andrea Mazzanti, ${ }^{11}$ Lodovico Lunazzi, ${ }^{1}$ Mirko Minzoni, ${ }^{1}$ and J. Edgar Anderson. ${ }^{* 2}$ \\ Department of Organic Chemistry "A. Mangini", University of Bologna, Viale \\ Risorgimento 4, 20196-Bologna, Italy, and Chemistry Department, University College, \\ Gower Street, London WC1E 6BT, UK.

\section{E-mail:mazzand@ms.fci.unibo.it; j.e.anderson@ucl.ac.uk}

Page S2-S4 Analytical and spectroscopic data for compounds 1a-1h, 5

Page S5 Ab-initio Computed Dihedral Angles for Compounds 1a-1h, 2a-2d, 5, and 6

Page S6-S10 Ab-initio computational data for compound 1a

Page S11-S14 Ab-initio computational data for compound $\mathbf{1 b}$

Page S15-S18 Ab-initio computational data for compound 1c

Page S19-S21 Ab-initio computational data for compound 1d

Page S22-S25 Ab-initio computational data for compound 1e

Page S26-S29 Ab-initio computational data for compound $1 \mathbf{f f}$

Page S30-S32 Ab-initio computational data for compound $\mathbf{1 h}$

Page S33-S34: Ab-initio computational data for compound 5 


\section{Analytical and Spectroscopic data for compounds 1a-1h and 5}

3'-Isopropyl-2-methyl-biphenyl (1a). HPLC eluent: $\mathrm{CH}_{3} \mathrm{CN} / \mathrm{H}_{2} \mathrm{O}$ 90:10 ${ }^{1} \mathrm{H}-\mathrm{NMR}$ $\left(600 \mathrm{MHz}, \mathrm{CDCl}_{3}, 25^{\circ} \mathrm{C}, \mathrm{TMS}\right): \delta=1.28(6 \mathrm{H}, \mathrm{d}, \mathrm{J}=6.9 \mathrm{~Hz}), 2.27(3 \mathrm{H}, \mathrm{s}), 2.94(1 \mathrm{H}$, septet, $\mathrm{J}=6.9 \mathrm{~Hz}), 7.13-7.33(8 \mathrm{H}, \mathrm{m}) .{ }^{13} \mathrm{C}-\mathrm{NMR}\left(150.8 \mathrm{MHz}, \mathrm{CDCl}_{3}, 25^{\circ} \mathrm{C}, \mathrm{TMS}\right)$ : $\delta=20.5\left(\mathrm{CH}_{3}\right), 24.1\left(\mathrm{CH}_{3}\right), 34.2(\mathrm{CH}), 124.9(\mathrm{CH}), 125.8(\mathrm{CH}), 126.7(\mathrm{CH}), 127.2(\mathrm{CH})$, $127.5(\mathrm{CH}), 128.0(\mathrm{CH}), 129.9(\mathrm{CH}), 130.3(\mathrm{CH}), 135.4(\mathrm{q}), 141.9$ (q), $142.3(\mathrm{q}), 148.6$ (q). Anal. Calcd for $\mathrm{C}_{16} \mathrm{H}_{18}$ C, 91.37; H, 8.63; found: C, 91.10; H, 8.85.

2-Ethyl-3'-isopropyl-biphenyl (1b). HPLC eluent: $\mathrm{CH}_{3} \mathrm{CN} / \mathrm{H}_{2} \mathrm{O}$ 90:10 ${ }^{1} \mathrm{H}-\mathrm{NMR}(600$ $\left.\mathrm{MHz}, \mathrm{CDCl}_{3}, 25^{\circ} \mathrm{C}, \mathrm{TMS}\right): \delta=0.88(3 \mathrm{H}, \mathrm{t}, \mathrm{J}=7.5 \mathrm{~Hz}), 1.05(6 \mathrm{H}, \mathrm{d}, \mathrm{J}=6.9 \mathrm{~Hz}), 2.39(2 \mathrm{H}$, q, J=7.5 Hz), $2.72\left(1 \mathrm{H}\right.$, septet, J=6.9 Hz), 6.50-7.32 (8H, m). ${ }^{13} \mathrm{C}-\mathrm{NMR}(150.8 \mathrm{MHz}$, $\left.\mathrm{CDCl}_{3}, 25^{\circ} \mathrm{C}, \mathrm{TMS}\right): \delta=14.6\left(\mathrm{CH}_{3}\right), 22.5\left(\mathrm{CH}_{3}\right), 25.3\left(\mathrm{CH}_{2}\right), 33.5(\mathrm{CH}), 124.1(\mathrm{CH})$, $124.7(\mathrm{CH}), 125.8(\mathrm{CH}), 126.6(\mathrm{CH}), 126.7(\mathrm{CH}), 127.1(\mathrm{CH}), 127.9(\mathrm{CH}), 129.2(\mathrm{CH})$, 141.4 (q), 141.6 (q), 141.7 (q), 148.3 (q). Anal. Calcd for $\mathrm{C}_{17} \mathrm{H}_{20} \mathrm{C}, 91.01 ; \mathrm{H}, 8.99$; found: C, 91.13; H, 8.82.

2,3'-Diisopropyl-biphenyl (1c). HPLC eluent: $\mathrm{CH}_{3} \mathrm{CN} / \mathrm{H}_{2} \mathrm{O}$ 90:10; ${ }^{1} \mathrm{H}-\mathrm{NMR}$ (600 $\left.\mathrm{MHz}, \mathrm{CDCl}_{3}, 25^{\circ} \mathrm{C}, \mathrm{TMS}\right): \delta=1.17(6 \mathrm{H}, \mathrm{d}, \mathrm{J}=6.7 \mathrm{~Hz}), 1.28(6 \mathrm{H}, \mathrm{d}, \mathrm{J}=6.9 \mathrm{~Hz}), 2.95(1 \mathrm{H}$, septet, J=6.9 Hz), $3.05(1 \mathrm{H}$, septet, $\mathrm{J}=6.9 \mathrm{~Hz}), 7.11-7.40(8 \mathrm{H}, \mathrm{m}) ;{ }^{13} \mathrm{C}-\mathrm{NMR}(150.8$ $\left.\mathrm{MHz}, \mathrm{CDCl}_{3}, 25^{\circ} \mathrm{C}, \mathrm{TMS}\right): \delta=23.6\left(\mathrm{CH}_{3}\right), 24.0\left(\mathrm{CH}_{3}\right), 29.0(\mathrm{CH}), 33.7(\mathrm{CH}), 124.4$ $(\mathrm{CH}), 124.8(\mathrm{CH}), 125.2(\mathrm{CH}), 126.3(\mathrm{CH}), 127.2(\mathrm{CH}), 127.5(\mathrm{CH}), 129.6(\mathrm{CH}), 141.0$ (q), 141.6 (q), 146.0 (q), 148.1 (q). Anal. Calcd for $\mathrm{C}_{18} \mathrm{H}_{22} \mathrm{C}, 90.70$; H, 9.30; found: $\mathrm{C}$, $91.01 ; \mathrm{H}, 9.18$.

2-tert-Butyl-3'-isopropyl-biphenyl (1d). HPLC eluent: $\mathrm{CH}_{3} \mathrm{CN} / \mathrm{H}_{2} \mathrm{O}$ 90:10; ${ }^{1} \mathrm{H}-\mathrm{NMR}$ (600 MHz, $\left.\mathrm{CDCl}_{3}, 25^{\circ} \mathrm{C}, \mathrm{TMS}\right): \delta=1.18(9 \mathrm{H}, \mathrm{s}), 1.26(6 \mathrm{H}, \mathrm{d}, \mathrm{J}=6.9 \mathrm{~Hz}), 1.54(3 \mathrm{H}, \mathrm{s})$, $2.91(1 \mathrm{H}$, septet, J=6.9 Hz), $7.04(1 \mathrm{H}, \mathrm{dd}, \mathrm{J}=7.2 \mathrm{~Hz}), 7.09(1 \mathrm{H}$, ddd, J=7.6 Hz), 7.14 $(1 \mathrm{H}, \mathrm{t}, \mathrm{J}=7.6 \mathrm{~Hz}), 7.16-7.18(2 \mathrm{H}, \mathrm{m}), 7.24(1 \mathrm{H}, \mathrm{s}), 7.25(1 \mathrm{H}, \mathrm{d}, \mathrm{J}=7.6 \mathrm{~Hz}), 7.30(1 \mathrm{H}$, ddd, J=8.1 Hz), $7.53(1 \mathrm{H}, \mathrm{dd}, \mathrm{J}=8.1 \mathrm{~Hz}) .{ }^{13} \mathrm{C}-\mathrm{NMR}\left(150.8 \mathrm{MHz}, \mathrm{CDCl}_{3}, 25^{\circ} \mathrm{C}, \mathrm{TMS}\right)$ : $\delta=24.2\left(\mathrm{CH}_{3}\right), 32.7\left(\mathrm{CH}_{3}\right), 34.2(\mathrm{CH}), 36.7(\mathrm{q}), 124.7(\mathrm{CH}), 125.0(\mathrm{CH}), 126.8(\mathrm{CH})$, $127.2(\mathrm{CH}), 127.6(\mathrm{CH}), 128.5(\mathrm{CH}), 132.6(\mathrm{CH}), 138.4(\mathrm{q}), 142.5(\mathrm{q}), 145.2(\mathrm{q}), 147.8$ (q). Anal. Calcd for $\mathrm{C}_{19} \mathrm{H}_{24} \mathrm{C}, 90.42 ; \mathrm{H}, 9.58$; found: C, 90.54; H,9.73. 
2-Chloro-3'-isopropyl-biphenyl (1e). HPLC eluent: $\mathrm{CH}_{3} \mathrm{CN} / \mathrm{H}_{2} \mathrm{O}$ 90:10; ${ }^{1} \mathrm{H}-\mathrm{NMR}$ $\left(600 \mathrm{MHz}, \mathrm{CDCl}_{3}, 25^{\circ} \mathrm{C}, \mathrm{TMS}\right): \delta=1.29(6 \mathrm{H}, \mathrm{d}, \mathrm{J}=6.6 \mathrm{~Hz}), 2.96(1 \mathrm{H}$, septet, $\mathrm{J}=6.6 \mathrm{~Hz})$, 7.23-7.48 (8H, m). ${ }^{13} \mathrm{C}-\mathrm{NMR}\left(150.8 \mathrm{MHz}, \mathrm{CDCl}_{3}, 25^{\circ} \mathrm{C}, \mathrm{TMS}\right): \delta=24.0\left(\mathrm{CH}_{3}\right), 34.1$ $(\mathrm{CH}), 125.7(\mathrm{CH}), 126.8(\mathrm{CH}), 126.85(\mathrm{CH}), 127.7(\mathrm{CH}), 127.9(\mathrm{CH}), 128.3(\mathrm{CH}) 129.9$ $(\mathrm{CH}), 131.4(\mathrm{CH}), 132.5$ (q), 139.3 (q), 140.8 (q), 148.6 (q). Anal. Calcd for $\mathrm{C}_{15} \mathrm{H}_{15} \mathrm{Cl}$ C, 78.08; H, 6.55; Cl, 15.37; found: C, 78.24; H, 6.67.

2-Bromo-3'-isopropyl-biphenyl (1f). HPLC eluent: $\mathrm{CH}_{3} \mathrm{CN} / \mathrm{H}_{2} \mathrm{O}$ 90:10; ${ }^{1} \mathrm{H}-\mathrm{NMR}$ (600 MHz, $\left.\mathrm{CDCl}_{3}, 25^{\circ} \mathrm{C}, \mathrm{TMS}\right): \delta=1.02(6 \mathrm{H}, \mathrm{d}, \mathrm{J}=6.8 \mathrm{~Hz}), 2.96(1 \mathrm{H}$, septet, $\mathrm{J}=6.8 \mathrm{~Hz})$, 7.17-7.67 (8H, m). ${ }^{13} \mathrm{C}-\mathrm{NMR}\left(150.8 \mathrm{MHz}, \mathrm{CDCl}_{3}, 25^{\circ} \mathrm{C}, \mathrm{TMS}\right): \delta=23.9\left(\mathrm{CH}_{3}\right), 34.0$ $(\mathrm{CH}), 122.7(\mathrm{q}), 125.7(\mathrm{CH}), 126.7(\mathrm{CH}), 127.3(\mathrm{CH}), 127.7(\mathrm{CH}), 127.8(\mathrm{CH}) 128.5$ $(\mathrm{CH}), 131.3(\mathrm{CH}), 133.1(\mathrm{CH}), 140.9$ (q), 142.8 (q), 148.4 (q). Anal. Calcd for $\mathrm{C}_{15} \mathrm{H}_{15} \mathrm{Br}$ C, 65.47; H, 5.49; Br, 29.04; found: C, 65.24; H, 5.25.

2-Iodo-3'-isopropyl-biphenyl (1g). HPLC eluent: $\mathrm{CH}_{3} \mathrm{CN} / \mathrm{H}_{2} \mathrm{O}$ 90:10; ${ }^{1} \mathrm{H}-\mathrm{NMR}$ (600 $\mathrm{MHz}, \mathrm{CDCl}_{3}, 25^{\circ} \mathrm{C}$, TMS): $\delta={ }^{13} \mathrm{C}-\mathrm{NMR}\left(150.8 \mathrm{MHz}, \mathrm{CDCl}_{3}, 25^{\circ} \mathrm{C}, \mathrm{TMS}\right): \delta=1.29$ $(6 \mathrm{H}, \mathrm{d}, \mathrm{J}=6.6 \mathrm{~Hz}), 2.96\left(1 \mathrm{H}\right.$, septet, J=6.6 Hz), 7.02-7.95 (8H, m). ${ }^{13} \mathrm{C}-\mathrm{NMR}(150.8$ $\left.\mathrm{MHz}, \mathrm{CDCl}_{3}, 25^{\circ} \mathrm{C}, \mathrm{TMS}\right): \delta=22.8\left(\mathrm{CH}_{3}\right), 33.8(\mathrm{CH}), 97.9(\mathrm{q}), 125.8(\mathrm{CH}), 126.4(\mathrm{CH})$, $126.4(\mathrm{CH}), 128.0(\mathrm{CH}), 128.1(\mathrm{CH}) 128.6(\mathrm{CH}), 130.0(\mathrm{CH}), 139.2(\mathrm{CH}), 143.7(\mathrm{q})$, 146.0 (q), 148.7 (q). Anal. Calcd for $\mathrm{C}_{15} \mathrm{H}_{15} \mathrm{I}$ C, 55.92; H, 4.69; I, 39.39; found: C, $55.74 ; \mathrm{H}, 4.77$.

3,3"'-Diisopropyl-[1,1';2',1'']terphenyl (1h). HPLC eluent: $\mathrm{CH}_{3} \mathrm{CN} / \mathrm{H}_{2} \mathrm{O}$ 90:10; ( ${ }^{1} \mathrm{H}-$ NMR $\left(600 \mathrm{MHz}, \mathrm{CDCl}_{3}, 25^{\circ} \mathrm{C}, \mathrm{TMS}\right): \delta=1.02(6 \mathrm{H}, \mathrm{d}, \mathrm{J}=6.9 \mathrm{~Hz}), 2.71(1 \mathrm{H}$, septet, $\mathrm{J}=6.9 \mathrm{~Hz}), 6.87-7.46(12 \mathrm{H}, \mathrm{m}) .{ }^{13} \mathrm{C}-\mathrm{NMR}\left(150.8 \mathrm{MHz}, \mathrm{CDCl}_{3}, 25^{\circ} \mathrm{C}, \mathrm{TMS}\right): \delta=24.0$ $\left(\mathrm{CH}_{3}\right), 34.0(\mathrm{CH}), 124.8(\mathrm{CH}), 127.2(\mathrm{CH}), 127.5(\mathrm{CH}), 128.0(\mathrm{CH}), 128.7(\mathrm{CH}), 130.5$ (CH), 141.3 (q), 141.6 (q), 148.3 (q). Anal. Calcd for $\mathrm{C}_{24} \mathrm{H}_{26} \mathrm{C}, 91.67$; H, 8.33; found: C, 91.10; H, 8.85.

5-Isopropyl-2,2'-dimethyl-biphenyl (5). this compound was obtained using the standard procedure from 2-methyl-phenylboronic acid and the mixture of 1-bromo-pcymene and 2-bromo-p-cymene. The two resulting biphenyls were separated by preparative HPLC (eluent: $\mathrm{CH}_{3} \mathrm{CN} / \mathrm{H}_{2} \mathrm{O}$ 80:20). ${ }^{1} \mathrm{H}-\mathrm{NMR}\left(600 \mathrm{MHz}, \mathrm{CDCl}_{3}, 2^{\circ} \mathrm{C}\right.$, TMS): $\delta=1.24(6 \mathrm{H}, \mathrm{d}, \mathrm{J}=7.0 \mathrm{~Hz}), 2.02(3 \mathrm{H}, \mathrm{s}), 2.06(3 \mathrm{H}, \mathrm{s}), 2.88$ (1H, septet, J=7.0), 6.87-7.46 (12H, m). ${ }^{13} \mathrm{C}-\mathrm{NMR}\left(150.8 \mathrm{MHz}, \mathrm{CDCl}_{3}, 25^{\circ} \mathrm{C}, \mathrm{TMS}\right): \delta=19.4\left(\mathrm{CH}_{3}\right), 20.0$ 
$\left(\mathrm{CH}_{3}\right), 24.1\left(\mathrm{CH}_{3} \mathrm{Ar}\right), 24.3\left(\mathrm{CH}_{3} \mathrm{Ar}\right), 33.8(\mathrm{CH}), 125.2(\mathrm{CH}), 125.6(\mathrm{CH}), 127.1(\mathrm{CH})$, $127.5(\mathrm{CH}), 129.5(\mathrm{CH}), 129.8(\mathrm{CH}), 129.9(\mathrm{CH}), 133.1$ (q), 136.0 (q), 141.5 (q), 142.1 (q), 146.2 (q). Anal. Calcd for $\mathrm{C}_{17} \mathrm{H}_{20} \mathrm{C}, 91.01 ; \mathrm{H}, 8.99$; found: C, 91.15; H, 8.83. 
Table . Ab-initio Computed Dihedral Angles for Compounds 1a-1h, 2a-2d, 5, and 6. Values Greater than $90^{\circ}$ Correspond to an anti-Conformer and those Smaller than $90^{\circ}$ to a syn- Conformer (see Scheme 1 and Figure 1).

\begin{tabular}{|c|c|c|c|}
\hline Compound & & $\begin{array}{c}\text { Angle of lower } \\
\text { ground state }\end{array}$ & $\begin{array}{l}\text { Angle of higher } \\
\text { ground state }\end{array}$ \\
\hline 2-methyl-3'-isopropyl-biphenyl & 1a & 127 & 45 \\
\hline 2-ethyl-3'-isopropyl-biphenyl & 1b & 118 & 67 \\
\hline 2,3'-di-isopropyl-biphenyl & 1c & 122 & 62 \\
\hline 2-t-butyl-3'-isopropyl-biphenyl & 1d & 95 & Not existing \\
\hline 2-chloro-3'-isopropyl-biphenyl & 1e & 56 & 126 \\
\hline 2-bromo-3'-isopropyl-biphenyl & 1f & 128 & 56 \\
\hline 2-iodo-3'-isopropyl-biphenyl & $1 \mathrm{~g}$ & Not calculated & Not calculated \\
\hline 3,3"-di-isopropyl-[1,1',2',1"]terphenyl & 1h & 53 (trans) & 131 and 51 (cis) \\
\hline 2,2"'-di-methyl[1,1',4',1'”]terphenyl & $2 a$ & $55(\operatorname{trans})^{\text {a) }}$ & 55 (cis) \\
\hline 2,2"'-di-ethyl[1,1',4',1']terphenyl & $2 \mathbf{b}$ & 64 (cis) & 64 (trans) \\
\hline 2,2"-di-isopropyl[1,1',4',1']terphenyl & 2c & 60 (trans) & 60 (cis) \\
\hline 2,2"'-di-tertbutyl[1,1',4',1']terphenyl & 2d & 93 (cis) & 93 (trans) $^{\text {a) }}$ \\
\hline 2,2'-di-methyl-5'-isopropylbiphenyl & 5 & 90 & Not existing \\
\hline 3,3',5,5'tetramethyl-biphenyl & & 39 & Not existing \\
\hline Biphenyl & & $38^{b)}$ & Not existing \\
\hline 3-isopropyl-biphenyl & & 38 & Not existing \\
\hline 2,4'-di-isopropyl-biphenyl & & 123 & 60 \\
\hline See compound $n^{\circ} 5$ of BFS (ref. 13) & 6 & 89 & Not existing \\
\hline
\end{tabular}

a) Experimental values for $\mathbf{2 a}$ and $\mathbf{2 d}$ in the crystal are $55^{\circ}$ and $99.5^{\circ}$ respectively. ${ }^{19}$

b) Calculations at a higher level ${ }^{22}$ suggest $\tau=43^{\circ}$. 
Compound 1a: ground state GS-1

B3LYP/6-31G(d) Energy: -620.56455732 a.u. Imaginary frequencies: 0

$\begin{array}{lrrr}\text { C } & 0.636046 & 0.448201 & 2.662152 \\ \mathrm{C} & 0.094660 & 0.567137 & 3.949267 \\ \mathrm{C} & -1.183518 & 0.104118 & 4.257463 \\ \mathrm{C} & -1.958845 & -0.486114 & 3.260443 \\ \mathrm{C} & -1.439800 & -0.609527 & 1.973658 \\ \mathrm{C} & -0.147758 & -0.159945 & 1.653986 \\ \mathrm{C} & 1.526429 & -1.009210 & -0.041061 \\ \mathrm{C} & 0.334062 & -0.331936 & 0.252075 \\ \mathrm{C} & -0.435234 & 0.152361 & -0.819064 \\ \mathrm{C} & -0.046434 & -0.013940 & -2.152653 \\ \mathrm{C} & 1.152346 & -0.693989 & -2.412154 \\ \mathrm{C} & 1.928462 & -1.187573 & -1.364642 \\ \mathrm{C} & 2.019763 & 1.001997 & 2.395867 \\ \mathrm{H} & 0.690623 & 1.045487 & 4.723644 \\ \mathrm{H} & -1.571728 & 0.211730 & 5.266934 \\ \mathrm{H} & -2.958797 & -0.850244 & 3.480896 \\ \mathrm{H} & -2.034234 & -1.079901 & 1.194794 \\ \mathrm{H} & 2.126619 & -1.417853 & 0.766539 \\ \mathrm{H} & -1.357178 & 0.686608 & -0.599353 \\ \mathrm{H} & 2.850563 & -1.721654 & -1.580171 \\ \mathrm{H} & 2.092725 & 1.457673 & 1.403768 \\ \mathrm{H} & 2.792344 & 0.224087 & 2.446638 \\ \mathrm{H} & 2.278243 & 1.760809 & 3.141722 \\ \mathrm{C} & -0.169054 & 1.637589 & -4.074403 \\ \mathrm{C} & -0.905491 & 0.540684 & -3.282196 \\ \mathrm{H} & 0.161538 & 2.448017 & -3.415705 \\ \mathrm{H} & 0.717183 & 1.235855 & -4.580117 \\ \mathrm{H} & -0.824541 & 2.066229 & -4.842000 \\ \mathrm{C} & -1.411868 & -0.571512 & -4.219743 \\ \mathrm{H} & -1.786147 & 1.005706 & -2.818481 \\ \mathrm{H} & -1.970549 & -1.333520 & -3.665483 \\ \mathrm{H} & -2.072064 & -0.155790 & -4.990357 \\ \mathrm{H} & -0.580447 & -1.072312 & -4.729906 \\ \mathrm{H} & 1.483248 & -0.840969 & -3.437340 \\ & & & \\ & & & \end{array}$


Compound 1a: ground state GS-2

B3LYP/6-31G(d) Energy: -620.56451503 a.u. Imaginary frequencies: $\odot$

\begin{tabular}{|c|c|c|c|}
\hline C & -2.28955600 & $-1.0017750 \odot$ & $-\odot .3432770 \odot$ \\
\hline C & -3.64570500 & $-1.2220950 \odot$ & $-\odot . \odot 67169 \odot \odot$ \\
\hline C & -4.43442500 & $-\odot .2629420 \odot$ & $\odot .5661230 \odot$ \\
\hline C & -3.87032800 & ๑.95902300 & $\odot .9297400 \odot$ \\
\hline C & -2.52489900 & 1.19863200 & $\odot .65967000$ \\
\hline C & -1.71622700 & ๑.23467700 & $\odot .0347500 \odot$ \\
\hline C & ๑. 05077600 & 1.73912800 & $-\odot .9216970 \odot$ \\
\hline C & $-\odot .28487200$ & 0.57129900 & $-\odot .2209240 \odot$ \\
\hline C & 0.75726400 & $-\odot .2365590 \odot$ & $\odot .2620430 \odot$ \\
\hline C & 2.10365900 & $\odot . \odot 852370 \odot$ & $\odot .0612050 \odot$ \\
\hline C & 2.40643700 & 1.25540000 & $-\odot .6509180 \odot$ \\
\hline C & 1.38798500 & 2.07382500 & -1.13598500 \\
\hline C & -1.50370100 & -2.07851400 & -1.06103100 \\
\hline $\mathrm{H}$ & -4.09267100 & -2.16725600 & $-\odot .36802100$ \\
\hline $\mathrm{H}$ & -5.48307000 & $-\odot .4673590 \odot$ & $\odot .7658530 \odot$ \\
\hline $\mathrm{H}$ & -4.47043800 & 1.72017200 & 1.42129200 \\
\hline $\mathrm{H}$ & $-2.0758940 \odot$ & 2.14471300 & $\odot .9504970 \odot$ \\
\hline $\mathrm{H}$ & -0.74037100 & 2.37423700 & -1.31102600 \\
\hline $\mathrm{H}$ & $\odot .5097320 \odot$ & -1.13012800 & $\odot .8300330 \odot$ \\
\hline $\mathrm{H}$ & 1.63631600 & 2.97702500 & -1.68780100 \\
\hline $\mathrm{H}$ & $-\odot .93935000$ & -2.71088200 & $-\odot .3636820 \odot$ \\
\hline $\mathrm{H}$ & $-\odot .7798090 \odot$ & -1.65482700 & -1.76341000 \\
\hline $\mathrm{H}$ & -2.17779300 & -2.73715400 & -1.61857700 \\
\hline C & 4.08540600 & -1.39817600 & $-\odot .5019790 \odot$ \\
\hline C & 3.20265200 & $-\odot .81233500$ & $\odot .61618600$ \\
\hline $\mathrm{H}$ & 3.48457400 & -1.94943500 & -1.23352500 \\
\hline $\mathrm{H}$ & 4.62406900 & $-\odot .6 \odot 87840 \odot$ & -1.03975300 \\
\hline $\mathrm{H}$ & 4.83188200 & $-2 . \odot 8478600$ & $-\odot . \odot 850810 \odot$ \\
\hline C & 4.05704700 & $-\odot . \odot 850360 \odot$ & 1.67160600 \\
\hline $\mathrm{H}$ & 2.70783600 & -1.65481700 & 1.11860900 \\
\hline $\mathrm{H}$ & 3.43513500 & $\odot .3015110 \odot$ & 2.48630400 \\
\hline $\mathrm{H}$ & 4.80153500 & $-\odot .76527700$ & 2.10254100 \\
\hline $\mathrm{H}$ & 4.59594600 & $0.7628290 \odot$ & 1.23225000 \\
\hline $\mathrm{H}$ & 3.4 & 1.53149500 & -08265 \\
\hline
\end{tabular}


Compound 1a: transition state TS-180

B3LYP/6-31G(d) Energy: -620.55323526 a.u. Imaginary frequencies: 1

\begin{tabular}{|c|c|c|c|}
\hline C & -2.84817400 & 0.60063600 & $\odot .0 \odot \odot \odot 38 ० \odot$ \\
\hline C & -4.07410700 & -0.08503900 & 0.00004600 \\
\hline C & -4.17102800 & -1.47110900 & 0.00001100 \\
\hline C & -2.99528700 & -2.21369800 & -0.00003400 \\
\hline C & -1.77072900 & -1.55775500 & -0.00004200 \\
\hline C & -1.63304300 & -0.15112900 & $-\odot .00001200$ \\
\hline C & ๑. 05300800 & 1.80091300 & -0.00010300 \\
\hline C & -0.23818300 & $\odot .42244300$ & -0.00003000 \\
\hline C & ๑. 88979200 & -0.43226600 & ๑. 00001500 \\
\hline C & 2.20985300 & ๑. 02046200 & $\odot .00000700$ \\
\hline C & 2.44513400 & 1.40171600 & -0.00006000 \\
\hline C & 1.36560200 & 2.27543100 & - ๑ . 00011900 \\
\hline C & -2.96700000 & 2.11238400 & ๑. . 0008600 \\
\hline $\mathrm{H}$ & -4.98706100 & ๑. 50536200 & ๑. .00008400 \\
\hline $\mathrm{H}$ & -5.14360800 & -1.95550800 & $\odot .00001400$ \\
\hline $\mathrm{H}$ & -3.02150400 & -3.30014000 & -0.00006700 \\
\hline $\mathrm{H}$ & -0.88953700 & -2.18422300 & -0.00008000 \\
\hline $\mathrm{H}$ & -0.73013600 & 2.53741900 & $-\odot .00015000$ \\
\hline $\mathrm{H}$ & $\odot .76020000$ & -1.50702600 & $\odot .00006200$ \\
\hline $\mathrm{H}$ & 1.53605400 & 3.34922900 & -0.00018100 \\
\hline $\mathrm{H}$ & -2.51079100 & 2.57036300 & $-\odot .88505600$ \\
\hline $\mathrm{H}$ & -2.51065100 & 2.57030000 & $\odot .88519000$ \\
\hline $\mathrm{H}$ & -4.02288600 & 2.39870100 & ๑. .00016900 \\
\hline C & 4.22459300 & -0.85787600 & -1.26969000 \\
\hline C & 3.36075300 & -0.97825600 & $\odot .00006800$ \\
\hline $\mathrm{H}$ & 3.61824000 & -0.97837100 & -2.17402900 \\
\hline $\mathrm{H}$ & 4.71712800 & 0.12025600 & -1.32600200 \\
\hline $\mathrm{H}$ & 5.00812100 & -1.62506400 & -1.27834800 \\
\hline C & 4.22464600 & -0.85765600 & 1.26977200 \\
\hline $\mathrm{H}$ & 2.91720600 & -1.98345900 & ๑. .00016800 \\
\hline $\mathrm{H}$ & 3.61832300 & -0.97797100 & 2.17415500 \\
\hline $\mathrm{H}$ & 5.00815700 & -1.62485900 & 1.27854200 \\
\hline $\mathrm{H}$ & 4.71720600 & $\odot .12047400$ & 1.32588400 \\
\hline $\mathrm{H}$ & 3.46056900 & 1.78930500 & -0.00007300 \\
\hline
\end{tabular}


Compound 1a: transition state TS- $\odot$

B3LYP/6-31G(d) Energy: -620.55308400 a.u. Imaginary frequencies: 1

\begin{tabular}{|c|c|c|c|}
\hline C & 2.22378400 & $-1.1210900 \odot$ & $-\odot . \odot \odot \odot \odot 210 \odot$ \\
\hline C & 3.61128800 & -1.34049200 & $\odot .0 \odot \odot \odot 34 \odot \odot$ \\
\hline C & 4.54517400 & -0.31183800 & $\odot .0 \odot \odot \odot 610 \odot$ \\
\hline C & 4.08094400 & ๑.99900100 & $\odot .0000210 \odot$ \\
\hline C & 2.71308100 & 1.24114300 & $-\odot . \odot \odot \odot \odot 25 \odot \odot$ \\
\hline C & 1.73436500 & ๑.22131800 & $-\odot .00 \odot \odot 330 \odot$ \\
\hline C & $-\odot .06961000$ & 2.00652800 & $-\odot . \odot \odot \odot \odot \odot 3 ० \odot$ \\
\hline C & ๑.28510100 & 0.63862600 & $-\odot .0000390 \odot$ \\
\hline C & $-\odot .7991680 \odot$ & $-\odot .2642770 \odot$ & $-\odot . \odot \odot \odot \odot 38 \odot \odot$ \\
\hline C & -2.14167400 & $\odot .13492900$ & $-\odot . \odot \odot \odot \odot 16 \odot \odot$ \\
\hline C & -2.43795000 & 1.50041900 & $\odot .0000100 \odot$ \\
\hline C & -1.39620000 & 2.42446500 & $\odot . \odot \odot \odot \odot 17 \odot \odot$ \\
\hline C & 1.38403500 & -2.38391400 & $-\odot . \odot \odot \odot \odot 73 \odot \odot$ \\
\hline $\mathrm{H}$ & 3.96311500 & -2.36921700 & $\odot .0000500 \odot$ \\
\hline $\mathrm{H}$ & $5.60890 \odot \odot \odot$ & $-\odot .5332450 \odot$ & $\odot .0 \odot \odot 106 \odot \odot$ \\
\hline $\mathrm{H}$ & 4.77366300 & 1.83635400 & $\odot . \odot \odot \odot \odot 26 \odot \odot$ \\
\hline $\mathrm{H}$ & 2.40797700 & $2.278440 \odot \odot$ & $-\odot . \odot \odot \odot \odot 63 \odot \odot$ \\
\hline $\mathrm{H}$ & $\odot .6855910 \odot$ & $2.7810000 \odot$ & $\odot .0 \odot \odot \odot 2 \odot \odot \odot$ \\
\hline $\mathrm{H}$ & $-\odot .6242050 \odot$ & $-1.3259400 \odot$ & $-\odot .0 \odot \odot \odot 54 \odot \odot$ \\
\hline $\mathrm{H}$ & -1.61515700 & 3.48937200 & $\odot . \odot \odot \odot \odot 4 \odot \odot \odot$ \\
\hline $\mathrm{H}$ & 0.74242000 & -2.46254200 & $-\odot .8851840 \odot$ \\
\hline $\mathrm{H}$ & $\odot .7424310 \odot$ & -2.46263200 & $\odot .88503900$ \\
\hline $\mathrm{H}$ & 2.03837800 & -3.26064800 & $-\odot .0001220 \odot$ \\
\hline C & -4.11495700 & $-\odot .83414300$ & 1.26957300 \\
\hline C & -3.24643000 & $-\odot .91540300$ & $-\odot . \odot \odot \odot \odot \odot 3 ० \odot$ \\
\hline $\mathrm{H}$ & -3.50406800 & $-\odot .92722400$ & 2.17411500 \\
\hline $\mathrm{H}$ & -4.65030600 & $\odot .12117500$ & 1.32548500 \\
\hline $\mathrm{H}$ & -4.86348200 & -1.63553200 & 1.27803900 \\
\hline C & -4.11508200 & $-\odot .83405800$ & -1.26949000 \\
\hline $\mathrm{H}$ & -2.75793400 & -1.89957500 & $-\odot . \odot \odot \odot \odot 6 \odot \odot \odot$ \\
\hline $\mathrm{H}$ & -3.50426000 & $-\odot .9268930 \odot$ & -2.17410100 \\
\hline $\mathrm{H}$ & -4.86348600 & -1.63556000 & -1.27802300 \\
\hline $\mathrm{H}$ & -4.65058700 & 0.12118600 & -1.32519400 \\
\hline $\mathrm{H}$ & -3.46919800 & 1 & - \\
\hline
\end{tabular}


Compound 1a: transition state TS-90

B3LYP/6-31G(d) Energy: -620.56363973 a.u. Imaginary frequencies: 1

\begin{tabular}{|c|c|c|c|}
\hline C & -2.44574300 & $\odot .89603700$ & $-\odot .2970480 \odot$ \\
\hline C & -3.77798300 & $\odot .70181000$ & $-\odot .68184900$ \\
\hline C & $-4.3691640 \odot$ & $-\odot .5617640 \odot$ & $-\odot .6548820 \odot$ \\
\hline C & -3.62524900 & -1.66350700 & $-\odot .2365530 \odot$ \\
\hline C & -2.29640400 & -1.48750500 & $\odot .15081800$ \\
\hline C & -1.69519200 & -0.22231800 & 0.12673200 \\
\hline C & $\odot .06 \odot 2510 \odot$ & 0.16082700 & 1.89440000 \\
\hline C & $-\odot .26659700$ & $-\odot .08180600$ & $\odot .55423500$ \\
\hline C & $\odot .77285900$ & $-\odot .20627800$ & -0.37934100 \\
\hline C & 2.11858100 & $-\odot .09428700$ & -0.01027800 \\
\hline C & $2.4162700 \odot$ & $\odot .15 \odot 4950 \odot$ & 1.33834900 \\
\hline C & 1.39678800 & $\odot .27584000$ & 2.28061800 \\
\hline C & -1.83504200 & 2.27872500 & -0.33973500 \\
\hline $\mathrm{H}$ & -4.36120800 & 1.56010100 & -1.00828600 \\
\hline $\mathrm{H}$ & -5.40571800 & $-\odot .6819700 \odot$ & $-\odot .9592090 \odot$ \\
\hline $\mathrm{H}$ & -4.07296800 & -2.65346700 & $-\odot .2102600 \odot$ \\
\hline $\mathrm{H}$ & -1.70857400 & -2.34092600 & $\odot .4791020 \odot$ \\
\hline $\mathrm{H}$ & $-0.7332180 \odot$ & $\odot .25452300$ & 2.63121000 \\
\hline $\mathrm{H}$ & $\odot .52175400$ & $-\odot .4008170 \odot$ & -1.42015200 \\
\hline $\mathrm{H}$ & 1.64375300 & 0.46210900 & 3.32288000 \\
\hline $\mathrm{H}$ & $-\odot .9791360 \odot$ & $2.32 \odot 970 \odot \odot$ & -1.02390900 \\
\hline $\mathrm{H}$ & -1.46194700 & 2.58650800 & $\odot .64406800$ \\
\hline $\mathrm{H}$ & -2.56844700 & 3.02051000 & -0.67107200 \\
\hline C & $4.039 \odot 20 \odot \odot$ & 1.05719800 & -1.20438700 \\
\hline C & 3.21931300 & $-\odot .2382970 \odot$ & -1.05378500 \\
\hline $\mathrm{H}$ & 3.39383400 & 1.90764600 & -1.45043400 \\
\hline $\mathrm{H}$ & 4.57320300 & 1.30080900 & -0.27823600 \\
\hline $\mathrm{H}$ & 4.78537100 & $\odot .95244500$ & $-2.0 \odot \odot 9850 \odot$ \\
\hline C & 4.13507800 & -1.44155900 & $-\odot .76014100$ \\
\hline $\mathrm{H}$ & 2.72646100 & $-\odot .42854200$ & -2.01695900 \\
\hline $\mathrm{H}$ & 3.55791600 & -2.36964800 & $-\odot .68597400$ \\
\hline $\mathrm{H}$ & 4.87958200 & -1.56455500 & -1.55589400 \\
\hline $\mathrm{H}$ & 4.67689600 & -1.30918900 & $\odot .18396600$ \\
\hline $\mathrm{H}$ & 3.45173600 & $\odot .2418330 \odot$ & 1.6572270 \\
\hline
\end{tabular}


Compound 1b: ground state GS-1

B3LYP/6-31G(d) Energy: -659.87714002 a.u. Imaginary frequencies: 01

$\begin{array}{lrrr}\mathrm{C} & 0.504189 & 0.005224 & -2.545966 \\ \mathrm{C} & 0.044765 & -0.298014 & -3.835255 \\ \mathrm{C} & -1.314483 & -0.374771 & -4.135073 \\ \mathrm{C} & -2.253232 & -0.150260 & -3.129179 \\ \mathrm{C} & -1.816666 & 0.156853 & -1.841796 \\ \mathrm{C} & -0.450388 & 0.246940 & -1.530921 \\ \mathrm{C} & 0.603679 & 1.784873 & 0.176840 \\ \mathrm{C} & -0.069786 & 0.592938 & -0.126898 \\ \mathrm{C} & -0.432560 & -0.255804 & 0.930797 \\ \mathrm{C} & -0.132806 & 0.045662 & 2.264660 \\ \mathrm{C} & 0.548041 & 1.241055 & 2.535504 \\ \mathrm{C} & 0.909741 & 2.102224 & 1.500224 \\ \mathrm{C} & 1.997820 & -0.025598 & -2.277619 \\ \mathrm{H} & 0.776346 & -0.484123 & -4.619106 \\ \mathrm{H} & -1.635831 & -0.608462 & -5.146785 \\ \mathrm{H} & -3.317145 & -0.204977 & -3.344200 \\ \mathrm{H} & -2.542683 & 0.349558 & -1.056117 \\ \mathrm{H} & 0.867652 & 2.471640 & -0.622896 \\ \mathrm{H} & -0.957514 & -1.180792 & 0.700990 \\ \mathrm{H} & 1.426854 & 3.031492 & 1.726000 \\ \mathrm{H} & 2.281769 & 0.752490 & -1.563425 \\ \mathrm{H} & 2.529530 & 0.197288 & -3.211047 \\ \mathrm{C} & 0.688156 & -1.472710 & 4.124603 \\ \mathrm{C} & -0.537349 & -0.909273 & 3.380792 \\ \mathrm{H} & 1.376375 & -1.971285 & 3.433364 \\ \mathrm{H} & 1.244955 & -0.677243 & 4.633973 \\ \mathrm{H} & 0.378676 & -2.201095 & 4.883744 \\ \mathrm{C} & -1.529149 & -0.257852 & 4.363044 \\ \mathrm{H} & -1.052553 & -1.756638 & 2.908140 \\ \mathrm{H} & -2.419665 & 0.109691 & 3.841598 \\ \mathrm{H} & -1.851276 & -0.980324 & 5.122439 \\ \mathrm{H} & -1.073549 & 0.592002 & 4.885030 \\ \mathrm{H} & 0.795801 & 1.505554 & 3.560601 \\ \mathrm{C} & 2.472421 & -1.388553 & -1.740630 \\ \mathrm{H} & 1.988568 & -1.622004 & -0.786238 \\ \mathrm{H} & 2.232476 & -2.193283 & -2.444797 \\ \mathrm{H} & 3.556861 & -1.387086 & -1.580021 \\ & & & \\ & & & \\ & & & \end{array}$


Compound 1b: ground state GS-2

B3LYP/6-31G(d) Energy: -659.87707772 a.u. Imaginary frequencies: 0

\begin{tabular}{|c|c|c|c|}
\hline $\mathrm{C}$ & $\odot .694566$ & -0.375534 & -2.256273 \\
\hline C & ๑.597077 & -0.800888 & -3.588608 \\
\hline C & $-\odot .629028$ & $-\odot .892484$ & -4.245276 \\
\hline C & -1.798361 & $-\odot .548909$ & -3.568134 \\
\hline C & -1.722697 & $-\odot .127470$ & -2.241942 \\
\hline C & $-\odot .493985$ & $-\odot .04 \odot 591$ & -1.567998 \\
\hline C & $-\odot .935571$ & 1.703928 & 0.188013 \\
\hline C & -0.503202 & 0.412439 & -0.142631 \\
\hline C & -0.134556 & $-\odot .455 \odot 25$ & $\odot .898367$ \\
\hline C & -0.175851 & -0.064825 & 2.241097 \\
\hline C & -0.602875 & 1.237849 & 2.539959 \\
\hline C & -0.981074 & 2.111506 & 1.522477 \\
\hline C & 2.071850 & -0.212383 & -1.640229 \\
\hline $\mathrm{H}$ & 1.509761 & -1.060750 & -4.121354 \\
\hline $\mathrm{H}$ & -0.669044 & -1.229895 & -5.277757 \\
\hline $\mathrm{H}$ & -2.763263 & $-\odot .614752$ & -4.063880 \\
\hline $\mathrm{H}$ & -2.631245 & $\odot .127555$ & -1.702466 \\
\hline $\mathrm{H}$ & -1.228891 & 2.388203 & -0.603685 \\
\hline $\mathrm{H}$ & 0.174623 & -1.468753 & 0.651949 \\
\hline $\mathrm{H}$ & -1.312969 & 3.117144 & 1.768369 \\
\hline H & 2.760867 & $-\odot .919601$ & -2.118719 \\
\hline $\mathrm{H}$ & 2.053265 & $-\odot .465122$ & -0.576271 \\
\hline C & 1.451159 & $-\odot .543188$ & 4.128825 \\
\hline C & $\odot .224552$ & -1.040522 & 3.340727 \\
\hline $\mathrm{H}$ & 2.302446 & $-\odot .361910$ & 3.463556 \\
\hline $\mathrm{H}$ & 1.233906 & ๑.393960 & 4.654970 \\
\hline $\mathrm{H}$ & 1.754951 & -1.283120 & 4.878963 \\
\hline C & -0.950747 & -1.354649 & 4.285493 \\
\hline $\mathrm{H}$ & $\odot .509846$ & -1.980551 & 2.848992 \\
\hline $\mathrm{H}$ & -1.812179 & -1.741637 & 3.730397 \\
\hline $\mathrm{H}$ & -0.658250 & -2.105194 & 5.029530 \\
\hline $\mathrm{H}$ & -1.277724 & $-\odot .459 \odot 91$ & 4.827198 \\
\hline $\mathrm{H}$ & $-\odot .644617$ & 1.571355 & 3.573988 \\
\hline C & 2.623588 & 1.216427 & -1.800782 \\
\hline $\mathrm{H}$ & 2.675003 & 1.501783 & -2.857665 \\
\hline $\mathrm{H}$ & 1.982938 & 1.941864 & -1.288004 \\
\hline $\mathrm{H}$ & 3.6315 & 1.294430 & 76 \\
\hline
\end{tabular}


Compound 1b: transition state TS-180

B3LYP/6-31G(d) Energy: -659.86344439 a.u. Imaginary frequencies: 1

$\begin{array}{lrrr}\text { C } & 2.186240 & -0.359073 & 1.396080 \\ \mathrm{C} & 1.410547 & -0.212245 & 2.538647 \\ \mathrm{C} & 0.028906 & -0.022455 & 2.408037 \\ \mathrm{C} & -0.523988 & 0.007788 & 1.126490 \\ \mathrm{C} & 0.227497 & -0.142753 & -0.063554 \\ \mathrm{C} & 1.611857 & -0.323190 & 0.124860 \\ \mathrm{C} & -2.623898 & 0.118136 & -2.583841 \\ \mathrm{C} & -1.860334 & 0.058260 & -1.425339 \\ \mathrm{C} & -0.455425 & -0.105299 & -1.411614 \\ \mathrm{C} & 0.174047 & -0.226931 & -2.690308 \\ \mathrm{C} & -0.622189 & -0.163952 & -3.847385 \\ \mathrm{C} & -1.999883 & 0.009458 & -3.821603 \\ \mathrm{C} & 1.660898 & -0.394728 & -2.978057 \\ \mathrm{C} & 2.458075 & 0.922065 & -2.911410 \\ \mathrm{H} & 3.260450 & -0.502395 & 1.483062 \\ \mathrm{H} & -1.594508 & 0.160771 & 1.081588 \\ \mathrm{H} & 2.279361 & -0.434493 & -0.710471 \\ \mathrm{H} & -3.700618 & 0.245811 & -2.510068 \\ \mathrm{H} & -2.400643 & 0.140703 & -0.492922 \\ \mathrm{H} & -0.124407 & -0.258578 & -4.809694 \\ \mathrm{H} & -2.568741 & 0.050444 & -4.746441 \\ \mathrm{H} & 2.112568 & -1.159651 & -2.340408 \\ \mathrm{H} & 2.067798 & 1.636210 & -3.644822 \\ \mathrm{H} & 2.393728 & 1.394549 & -1.926467 \\ \mathrm{H} & 3.516707 & 0.748122 & -3.137933 \\ \mathrm{H} & 1.755347 & -0.790078 & -3.995985 \\ \mathrm{H} & 1.875321 & -0.240593 & 3.520867 \\ \mathrm{C} & -0.863636 & 0.150845 & 3.631264 \\ \mathrm{H} & -1.891668 & 0.282229 & 3.266768 \\ \mathrm{C} & -0.495742 & 1.412766 & 4.434314 \\ \mathrm{H} & -1.183484 & 1.548044 & 5.277645 \\ \mathrm{H} & 0.519991 & 1.343483 & 4.841541 \\ \mathrm{H} & -0.542995 & 2.309553 & 3.807009 \\ \mathrm{C} & -0.851264 & -1.099269 & 4.531085 \\ \mathrm{H} & 0.147359 & -1.282068 & 4.945465 \\ \mathrm{H} & -1.542661 & -0.975647 & 5.373240 \\ \mathrm{H} & -1.149162 & -1.992961 & 3.972031 \\ & & & \\ & & & \\ & & & \end{array}$


Compound 1b: transition state TS- $\odot$

B3LYP/6-31G(d) Energy: -659.86322511 a.u. Imaginary frequencies: 1

$\begin{array}{lrrr}\mathrm{C} & -2.547984 & -0.295217 & -1.534209 \\ \mathrm{C} & -1.607784 & -0.159770 & -2.551756 \\ \mathrm{C} & -0.265849 & 0.042573 & -2.219053 \\ \mathrm{C} & 0.091205 & 0.106604 & -0.866460 \\ \mathrm{C} & -0.829066 & -0.017511 & 0.195575 \\ \mathrm{C} & -2.170402 & -0.227020 & -0.196881 \\ \mathrm{C} & -1.334376 & -0.004842 & 3.974348 \\ \mathrm{C} & -1.512406 & -0.053306 & 2.597989 \\ \mathrm{C} & -0.460436 & 0.062686 & 1.659579 \\ \mathrm{C} & 0.848004 & 0.254247 & 2.204932 \\ \mathrm{C} & 1.000254 & 0.301767 & 3.601975 \\ \mathrm{C} & -0.056722 & 0.172805 & 4.493457 \\ \mathrm{C} & 2.159933 & 0.388787 & 1.441460 \\ \mathrm{C} & 2.743129 & -0.957238 & 0.970388 \\ \mathrm{H} & -3.594435 & -0.458222 & -1.779794 \\ \mathrm{H} & 1.137233 & 0.256051 & -0.664214 \\ \mathrm{H} & -2.957378 & -0.346183 & 0.535187 \\ \mathrm{H} & -2.195520 & -0.102476 & 4.630049 \\ \mathrm{H} & -2.527981 & -0.184152 & 2.252071 \\ \mathrm{H} & 2.002943 & 0.448610 & 3.996749 \\ \mathrm{H} & 0.116220 & 0.217494 & 5.565229 \\ \mathrm{H} & 2.073440 & 1.091699 & 0.608325 \\ \mathrm{H} & 2.938927 & -1.604901 & 1.831961 \\ \mathrm{H} & 2.058986 & -1.493040 & 0.305358 \\ \mathrm{H} & 3.689496 & -0.806408 & 0.437504 \\ \mathrm{H} & 2.888418 & 0.848723 & 2.119000 \\ \mathrm{H} & -1.919216 & -0.215533 & -3.591610 \\ \mathrm{C} & 0.803642 & 0.190388 & -3.294949 \\ \mathrm{H} & 1.764091 & 0.330864 & -2.780339 \\ \mathrm{C} & 0.566050 & 1.434866 & -4.171286 \\ \mathrm{H} & 1.378173 & 1.557621 & -4.897985 \\ \mathrm{H} & -0.372396 & 1.353528 & -4.732744 \\ \mathrm{H} & 0.512020 & 2.343777 & -3.562192 \\ \mathrm{C} & 0.927523 & -1.078606 & -4.159257 \\ \mathrm{H} & 0.004546 & -1.271327 & -4.719109 \\ \mathrm{H} & 1.741377 & -0.973508 & -4.886763 \\ \mathrm{H} & 1.132762 & -1.959895 & -3.541799\end{array}$


Compound 1c: ground state GS-1

B3LYP/6-31G(d) Energy: -699.18944590 a.u. Imaginary frequencies: 0

$\begin{array}{lrrr}\mathrm{C} & -0.016248 & 0.133129 & -2.325994 \\ \mathrm{C} & 0.675879 & 0.330722 & -3.531124 \\ \mathrm{C} & 2.055073 & 0.164998 & -3.629035 \\ \mathrm{C} & 2.785686 & -0.201245 & -2.498736 \\ \mathrm{C} & 2.119587 & -0.406099 & -1.294160 \\ \mathrm{C} & 0.725232 & -0.256120 & -1.185835 \\ \mathrm{C} & -0.856716 & -1.521685 & 0.331597 \\ \mathrm{C} & 0.106096 & -0.518149 & 0.149081 \\ \mathrm{C} & 0.528110 & 0.210210 & 1.272901 \\ \mathrm{C} & 0.013783 & -0.027123 & 2.552538 \\ \mathrm{C} & -0.953079 & -1.032074 & 2.700780 \\ \mathrm{C} & -1.381112 & -1.772359 & 1.599653 \\ \mathrm{C} & -1.521682 & 0.389404 & -2.286799 \\ \mathrm{H} & 0.119370 & 0.632072 & -4.415188 \\ \mathrm{H} & 2.555290 & 0.325279 & -4.580681 \\ \mathrm{H} & 3.862946 & -0.333396 & -2.555211 \\ \mathrm{H} & 2.678694 & -0.708834 & -0.412693 \\ \mathrm{H} & -1.179686 & -2.117320 & -0.517838 \\ \mathrm{H} & 1.272827 & 0.991796 & 1.137820 \\ \mathrm{H} & -2.123366 & -2.555868 & 1.730497 \\ \mathrm{H} & -1.862053 & 0.239689 & -1.258226 \\ \mathrm{C} & -0.638754 & 1.639696 & 4.351302 \\ \mathrm{C} & 0.495717 & 0.794303 & 3.741701 \\ \mathrm{H} & -1.090897 & 2.296448 & 3.600099 \\ \mathrm{H} & -1.433301 & 1.004462 & 4.760696 \\ \mathrm{H} & -0.258770 & 2.265122 & 5.168011 \\ \mathrm{C} & 1.168762 & -0.083396 & 4.813339 \\ \mathrm{H} & 1.256295 & 1.491323 & 3.363959 \\ \mathrm{H} & 1.997673 & -0.660621 & 4.389413 \\ \mathrm{H} & 1.564490 & 0.536305 & 5.626947 \\ \mathrm{H} & 0.458034 & -0.793381 & 5.252724 \\ \mathrm{H} & -1.373701 & -1.242284 & 3.681106 \\ \mathrm{C} & -2.296836 & -0.600695 & -3.177304 \\ \mathrm{H} & -2.020491 & -0.492132 & -4.232930 \\ \mathrm{H} & -2.097141 & -1.639028 & -2.889368 \\ \mathrm{H} & -3.376687 & -0.427102 & -3.096560 \\ \mathrm{C} & -1.859300 & 1.845707 & -2.663406 \\ \mathrm{H} & -1.578668 & 2.073033 & -3.698532 \\ \mathrm{H} & -2.936665 & 2.025257 & -2.564851 \\ \mathrm{H} & -1.335389 & 2.553830 & -2.012198 \\ & & & \\ & & & \end{array}$


Compound 1c: ground state GS-2

B3LYP/6-31G(d) Energy: -699.18936800 a.u. Imaginary frequencies: 0

$\begin{array}{lrrr}\mathrm{C} & 0.384400 & -0.057862 & -2.157333 \\ \mathrm{C} & 0.315285 & -0.477681 & -3.494939 \\ \mathrm{C} & -0.876438 & -0.903354 & -4.076293 \\ \mathrm{C} & -2.047675 & -0.910042 & -3.318822 \\ \mathrm{C} & -2.001288 & -0.502542 & -1.988856 \\ \mathrm{C} & -0.801023 & -0.085230 & -1.386364 \\ \mathrm{C} & -1.685676 & 1.363432 & 0.466005 \\ \mathrm{C} & -0.854135 & 0.312849 & 0.053682 \\ \mathrm{C} & -0.127236 & -0.384212 & 1.032814 \\ \mathrm{C} & -0.201780 & -0.055670 & 2.390643 \\ \mathrm{C} & -1.035705 & 1.006739 & 2.770203 \\ \mathrm{C} & -1.771077 & 1.707007 & 1.815803 \\ \mathrm{C} & 1.715121 & 0.452293 & -1.607694 \\ \mathrm{H} & 1.218528 & -0.461730 & -4.099869 \\ \mathrm{H} & -0.890277 & -1.222864 & -5.115179 \\ \mathrm{H} & -2.987485 & -1.236111 & -3.756527 \\ \mathrm{H} & -2.905533 & -0.521049 & -1.386155 \\ \mathrm{H} & -2.255281 & 1.916690 & -0.275886 \\ \mathrm{H} & 0.500518 & -1.217971 & 0.725470 \\ \mathrm{H} & -2.413776 & 2.528021 & 2.123774 \\ \mathrm{H} & 1.551012 & 0.772583 & -0.574789 \\ \mathrm{C} & 1.650626 & 0.042115 & 4.121201 \\ \mathrm{C} & 0.601963 & -0.841540 & 3.419174 \\ \mathrm{H} & 2.323295 & 0.511314 & 3.394936 \\ \mathrm{H} & 1.174536 & 0.842457 & 4.700191 \\ \mathrm{H} & 2.257232 & -0.553659 & 4.813876 \\ \mathrm{C} & -0.305795 & -1.544149 & 4.445980 \\ \mathrm{H} & 1.146036 & -1.623901 & 2.872542 \\ \mathrm{H} & -1.030795 & -2.199352 & 3.950982 \\ \mathrm{H} & 0.290472 & -2.153313 & 5.135755 \\ \mathrm{H} & -0.867015 & -0.817327 & 5.045235 \\ \mathrm{H} & -1.114719 & 1.288226 & 3.817340 \\ \mathrm{C} & 2.212995 & 1.686240 & -2.386183 \\ \mathrm{H} & 2.432874 & 1.444627 & -3.432820 \\ \mathrm{H} & 1.464692 & 2.486207 & -2.378359 \\ \mathrm{H} & 3.133497 & 2.076940 & -1.935745 \\ \mathrm{C} & 2.789254 & -0.651784 & -1.582080 \\ \mathrm{H} & 3.020771 & -1.010819 & -2.591976 \\ \mathrm{H} & 3.719931 & -0.273657 & -1.142129 \\ \mathrm{H} & 2.460597 & -1.514973 & -0.992309 \\ & & & \\ & & & \end{array}$


Compound 1c: transition state TS-180

B3LYP/6-31G(d) Energy: -699.17169170 a.u. Imaginary frequencies: 1

$\begin{array}{lrrr}\mathrm{C} & 0.139265 & -0.076983 & -2.442070 \\ \mathrm{C} & 1.048849 & 0.017870 & -3.508876 \\ \mathrm{C} & 2.425560 & 0.104441 & -3.342700 \\ \mathrm{C} & 2.934927 & 0.079683 & -2.051095 \\ \mathrm{C} & 2.058568 & -0.001024 & -0.977250 \\ \mathrm{C} & 0.650487 & -0.057335 & -1.101019 \\ \mathrm{C} & -1.548759 & -0.184266 & 0.282142 \\ \mathrm{C} & -0.145025 & -0.087647 & 0.190626 \\ \mathrm{C} & 0.522183 & -0.003873 & 1.437919 \\ \mathrm{C} & -0.120001 & -0.034817 & 2.676797 \\ \mathrm{C} & -1.515183 & -0.151895 & 2.707484 \\ \mathrm{C} & -2.212831 & -0.219948 & 1.509380 \\ \mathrm{C} & -1.342092 & -0.169539 & -2.834532 \\ \mathrm{H} & 0.660341 & 0.017846 & -4.520222 \\ \mathrm{H} & 3.079879 & 0.175854 & -4.207379 \\ \mathrm{H} & 4.005416 & 0.121726 & -1.868567 \\ \mathrm{H} & 2.512767 & -0.029411 & 0.002253 \\ \mathrm{H} & -2.168567 & -0.225271 & -0.594483 \\ \mathrm{H} & 1.597764 & 0.103117 & 1.476970 \\ \mathrm{H} & -3.297160 & -0.298984 & 1.515099 \\ \mathrm{H} & -1.827685 & -0.864688 & -2.146817 \\ \mathrm{C} & 0.321760 & 1.308221 & 4.785583 \\ \mathrm{C} & 0.693986 & 0.061264 & 3.961695 \\ \mathrm{H} & 0.439738 & 2.223222 & 4.194978 \\ \mathrm{H} & -0.718958 & 1.263778 & 5.128091 \\ \mathrm{H} & 0.960257 & 1.389248 & 5.673439 \\ \mathrm{C} & 0.573471 & -1.218250 & 4.811227 \\ \mathrm{H} & 1.748547 & 0.163186 & 3.670832 \\ \mathrm{H} & 0.875699 & -2.102870 & 4.240157 \\ \mathrm{H} & 1.209254 & -1.152229 & 5.702322 \\ \mathrm{H} & -0.457575 & -1.375945 & 5.149731 \\ \mathrm{H} & -2.049091 & -0.179958 & 3.653862 \\ \mathrm{C} & -1.608701 & -0.769546 & -4.229492 \\ \mathrm{H} & -1.332785 & -0.090498 & -5.043972 \\ \mathrm{H} & -1.074601 & -1.714583 & -4.375526 \\ \mathrm{H} & -2.681566 & -0.968876 & -4.332561 \\ \mathrm{C} & -2.025366 & 1.211779 & -2.734822 \\ \mathrm{H} & -1.590573 & 1.893480 & -3.475409 \\ \mathrm{H} & -3.100438 & 1.133264 & -2.939015 \\ \mathrm{H} & -1.897243 & 1.669773 & -1.749763 \\ & & & \\ & & & \end{array}$


Compound 1c: transition state TS- $\odot$

B3LYP/6-31G(d) Energy: -699.17150883 a.u. Imaginary frequencies: 1

$\begin{array}{lrrr}\text { C } & 0.474289 & -0.079767 & 2.151303 \\ \mathrm{C} & 0.470453 & -0.038101 & 3.555907 \\ \mathrm{C} & -0.687419 & 0.001873 & 4.322770 \\ \mathrm{C} & -1.911428 & -0.017339 & 3.667027 \\ \mathrm{C} & -1.937575 & -0.043865 & 2.279240 \\ \mathrm{C} & -0.783510 & -0.049127 & 1.460900 \\ \mathrm{C} & -2.334122 & 0.072161 & -0.549050 \\ \mathrm{C} & -1.018198 & -0.018742 & -0.037878 \\ \mathrm{C} & -0.007298 & -0.062185 & -1.023093 \\ \mathrm{C} & -0.255154 & -0.046946 & -2.401770 \\ \mathrm{C} & -1.576281 & 0.025111 & -2.849004 \\ \mathrm{C} & -2.604451 & 0.090562 & -1.913402 \\ \mathrm{H} & 1.858399 & -0.133403 & 1.489535 \\ \mathrm{H} & 1.421333 & -0.044790 & 4.075417 \\ \mathrm{H} & -0.627269 & 0.032640 & 5.407348 \\ \mathrm{H} & -2.845957 & -0.012313 & 4.221689 \\ \mathrm{H} & -2.917482 & -0.071022 & 1.825566 \\ \mathrm{H} & -3.188515 & 0.143928 & 0.108889 \\ \mathrm{H} & 1.029996 & -0.100413 & -0.742102 \\ \mathrm{H} & -3.637494 & 0.160661 & -2.244888 \\ \mathrm{C} & 1.788967 & -0.793486 & 0.622613 \\ \mathrm{C} & 0.859928 & -1.375596 & -4.253877 \\ \mathrm{H} & 0.910039 & -0.105988 & -3.383102 \\ \mathrm{H} & 0.840781 & -2.279663 & -3.635543 \\ \mathrm{H} & -0.033761 & -1.387104 & -4.889124 \\ \mathrm{C} & 1.735960 & -1.428726 & -4.911394 \\ \mathrm{H} & 0.989470 & 1.160296 & -4.256587 \\ \mathrm{H} & 1.833152 & -0.152374 & -2.789241 \\ \mathrm{H} & 1.063673 & 2.062678 & -3.639948 \\ \mathrm{H} & 1.866128 & 1.122282 & -4.914382 \\ \mathrm{H} & 0.101689 & 1.262587 & -4.892009 \\ \mathrm{C} & -1.803432 & 0.038999 & -3.911831 \\ \mathrm{H} & 2.968056 & -0.762614 & 2.355202 \\ \mathrm{H} & 3.281987 & -0.115421 & 3.181786 \\ \mathrm{H} & 2.659931 & -1.726914 & 2.773318 \\ \mathrm{C} & 3.853481 & -0.932561 & 1.731823 \\ \mathrm{H} & 2.303451 & 1.271651 & 1.028457 \\ \mathrm{H} & 2.447035 & 1.919585 & 1.901254 \\ \mathrm{H} & 3.254949 & 1.223385 & 0.484277 \\ & 1.562942 & 1.750169 & 0.381006\end{array}$


Compound 1d: ground state GS-1

B3LYP/6-31G(d) Energy: - 738.49115900 a.u. Imaginary frequencies: 0

\begin{tabular}{|c|c|c|c|}
\hline$C$ & -0.001478 & -0.176484 & 2.255563 \\
\hline C & 0.593425 & -0.570654 & 3.467925 \\
\hline C & 1.972169 & -0.682550 & 3.641871 \\
\hline C & 2.825137 & -0.397535 & 2.582385 \\
\hline C & 2.269329 & $-\odot .005028$ & 1.368298 \\
\hline C & ๑.881659 & 0.113370 & 1.177565 \\
\hline C & $\odot .420524$ & 1.911995 & -0.539395 \\
\hline C & 0.498434 & 0.552136 & -0.210730 \\
\hline C & $\odot .365777$ & -0.389362 & -1.243047 \\
\hline C & $\odot .124080$ & -0.013202 & -2.569866 \\
\hline C & $\odot .026555$ & 1.354861 & -2.862540 \\
\hline C & $\odot .178460$ & 2.307138 & -1.856447 \\
\hline C & -1.549657 & -0.085050 & 2.169013 \\
\hline $\mathrm{H}$ & $-\odot .034755$ & -0.803529 & 4.317833 \\
\hline $\mathrm{H}$ & 2.368105 & -0.992480 & 4.605664 \\
\hline $\mathrm{H}$ & 3.903357 & -0.477150 & 2.692306 \\
\hline $\mathrm{H}$ & 2.920948 & 0.222420 & $\odot .528747$ \\
\hline $\mathrm{H}$ & $\odot .555261$ & 2.659215 & $\odot .237763$ \\
\hline $\mathrm{H}$ & $\odot .457881$ & -1.446020 & -1.001331 \\
\hline $\mathrm{H}$ & 0.115569 & 3.365098 & -2.098655 \\
\hline C & -1.422949 & -1.056033 & -4.289385 \\
\hline C & $-\odot .018066$ & -1.070850 & -3.657331 \\
\hline $\mathrm{H}$ & -2.200806 & -1.188850 & -3.529535 \\
\hline H & -1.617721 & -0.107677 & -4.804356 \\
\hline H & -1.523903 & -1.861635 & -5.026493 \\
\hline C & 1.074062 & -0.937040 & -4.735204 \\
\hline $\mathrm{H}$ & $\odot .114360$ & -2.048768 & -3.174861 \\
\hline H & 2.075257 & $-\odot .981890$ & -4.292944 \\
\hline $\mathrm{H}$ & $\odot .989852$ & -1.744069 & -5.472884 \\
\hline $\mathrm{H}$ & $\odot .988720$ & 0.014755 & -5.272899 \\
\hline H & -0.160126 & 1.680478 & -3.882918 \\
\hline C & -1.999828 & 1.360628 & 1.848750 \\
\hline $\mathrm{H}$ & -1.604975 & 2.065085 & 2.590488 \\
\hline $\mathrm{H}$ & -1.673956 & 1.687518 & $\odot .860134$ \\
\hline $\mathrm{H}$ & -3.094872 & 1.423050 & 1.875790 \\
\hline C & -2.230489 & -0.473110 & 3.501470 \\
\hline $\mathrm{H}$ & -1.945199 & $\odot .192663$ & 4.323698 \\
\hline $\mathrm{H}$ & -3.316632 & -0.395222 & 3.381137 \\
\hline $\mathrm{H}$ & -2.007771 & -1.504239 & 3.797929 \\
\hline C & -2.100167 & -1.052891 & 1.094030 \\
\hline $\mathrm{H}$ & -3.196915 & -1.023635 & 1.094398 \\
\hline $\mathrm{H}$ & -1.761806 & -0.793028 & $\odot . \odot 899 \odot 7$ \\
\hline $\mathrm{H}$ & 7917 & -2.084126 & 1 \\
\hline
\end{tabular}


Compound 1d: transition state TS-180

B3LYP/6-31G(d) Energy: -738.46673300 a.u. Imaginary frequencies: 1

$\begin{array}{lrrr}\mathrm{C} & 0.352161 & 0.000123 & 2.323399 \\ \mathrm{C} & 1.353664 & 0.000392 & 3.311549 \\ \mathrm{C} & 2.718853 & 0.000320 & 3.051380 \\ \mathrm{C} & 3.132589 & -0.000119 & 1.729608 \\ \mathrm{C} & 2.173007 & -0.000336 & 0.727053 \\ \mathrm{C} & 0.773726 & -0.000155 & 0.939470 \\ \mathrm{C} & -1.480832 & -0.000285 & -0.390711 \\ \mathrm{C} & -0.071908 & -0.000114 & -0.332761 \\ \mathrm{C} & 0.556194 & 0.000082 & -1.605219 \\ \mathrm{C} & -0.123505 & 0.000079 & -2.824454 \\ \mathrm{C} & -1.523343 & -0.000076 & -2.815954 \\ \mathrm{C} & -2.184578 & -0.000267 & -1.596436 \\ \mathrm{C} & -1.109897 & -0.000014 & 2.851722 \\ \mathrm{H} & 1.058057 & 0.000630 & 4.350720 \\ \mathrm{H} & 3.431746 & 0.000542 & 3.871563 \\ \mathrm{H} & 4.186639 & -0.000292 & 1.465112 \\ \mathrm{H} & 2.560394 & -0.000720 & -0.279287 \\ \mathrm{H} & -2.077334 & -0.000545 & 0.499958 \\ \mathrm{H} & 1.633388 & 0.000275 & -1.689924 \\ \mathrm{H} & -3.271349 & -0.000441 & -1.565275 \\ \mathrm{C} & 0.387082 & -1.269708 & -4.961236 \\ \mathrm{C} & 0.658481 & 0.000278 & -4.133032 \\ \mathrm{H} & 0.617204 & -2.173687 & -4.386884 \\ \mathrm{H} & -0.664154 & -1.328054 & -5.267690 \\ \mathrm{H} & 0.999295 & -1.277148 & -5.871057 \\ \mathrm{C} & 0.386069 & 1.269839 & -4.961549 \\ \mathrm{H} & 1.726129 & 0.000746 & -3.873644 \\ \mathrm{H} & 0.615411 & 2.174144 & -4.387400 \\ \mathrm{H} & 0.998339 & 1.277566 & -5.871328 \\ \mathrm{H} & -0.665188 & 1.327254 & -5.268106 \\ \mathrm{H} & -2.085760 & -0.000045 & -3.746135 \\ \mathrm{C} & -1.824595 & 1.322025 & 2.435658 \\ \mathrm{H} & -1.503021 & 2.126052 & 3.107119 \\ \mathrm{H} & -1.602663 & 1.657875 & 1.423695 \\ \mathrm{H} & -2.913288 & 1.225621 & 2.535549 \\ \mathrm{C} & -1.216586 & 0.000045 & 4.400409 \\ \mathrm{H} & -0.768989 & 0.889648 & 4.855508 \\ \mathrm{H} & -2.280005 & 0.000161 & 4.667895 \\ \mathrm{H} & -0.769127 & -0.889603 & 4.855570 \\ \mathrm{C} & -1.824453 & -1.322114 & 2.435691 \\ \mathrm{H} & -1.502638 & -2.126166 & 3.107019 \\ \mathrm{H} & -2.913143 & -1.225871 & 2.535836 \\ \mathrm{H} & -1.602724 & -1.657904 & 1.423667\end{array}$


Compound 1d: transition state TS- $\odot$

B3LYP/6-31G(d) Energy: -738.46643064 a.u. Imaginary frequencies: 1

\begin{tabular}{|c|c|c|c|}
\hline C & -0.310865 & $-\odot . \odot \odot \odot \odot \odot 8$ & 2.157998 \\
\hline C & -0.203536 & $\odot .00 \odot \odot 76$ & 3.560802 \\
\hline C & $\odot .997044$ & $\odot .0 \odot \odot 194$ & 4.260629 \\
\hline C & 2.176785 & $\odot .0 \odot \odot 2 \odot 5$ & 3.534901 \\
\hline C & 2.110726 & 0.000128 & 2.148746 \\
\hline C & $\odot .915622$ & $\odot .0 \odot \odot \odot 58$ & 1.390522 \\
\hline C & 2.426323 & $-\odot .0 \odot \odot 108$ & $-\odot .667538$ \\
\hline C & 1.117998 & $\odot .000 \odot 27$ & -0.123971 \\
\hline C & ๑. 094033 & $\odot .0 \odot \odot \odot 14$ & -1.096588 \\
\hline C & 0.314173 & $-\odot . \odot \odot \odot \odot 92$ & -2.480515 \\
\hline C & 1.626690 & $-\odot .0 \odot \odot 2 \odot 5$ & -2.955047 \\
\hline C & 2.671756 & $-\odot .0 \odot \odot 214$ & -2.036793 \\
\hline C & -1.761283 & $-\odot .0 \odot \odot 164$ & 1.598840 \\
\hline $\mathrm{H}$ & -1.108529 & $\odot . ๑ \odot \odot \odot \odot 5$ & 4.150721 \\
\hline $\mathrm{H}$ & ๑.996595 & $\odot .000230$ & 5.347322 \\
\hline $\mathrm{H}$ & 3.145654 & ๑. 000307 & 4.027093 \\
\hline $\mathrm{H}$ & 3.063500 & $\odot .000154$ & 1.643530 \\
\hline $\mathrm{H}$ & 3.302916 & $-\odot .00 \odot 145$ & -0.037035 \\
\hline $\mathrm{H}$ & $-\odot .936991$ & $\odot .00 \odot 106$ & $-\odot .800969$ \\
\hline $\mathrm{H}$ & 3.701753 & -0.000315 & -2.384756 \\
\hline C & $-\odot .908178$ & 1.269771 & -4.307107 \\
\hline C & $-\odot .874169$ & $\odot .0 \odot \odot \odot \odot 7$ & -3.435691 \\
\hline $\mathrm{H}$ & $-\odot .921732$ & 2.174191 & -3.689144 \\
\hline H & $-\odot .03 \odot 756$ & 1.326427 & -4.962351 \\
\hline H & -1.800201 & 1.277576 & -4.944979 \\
\hline C & $-\odot .907785$ & -1.269113 & -4.308049 \\
\hline $\mathrm{H}$ & -1.785052 & $-\odot .0 \odot \odot 368$ & -2.821689 \\
\hline H & -0.920852 & -2.174006 & -3.690769 \\
\hline $\mathrm{H}$ & -1.799923 & -1.276801 & -4.945758 \\
\hline $\mathrm{H}$ & $-\odot .030483$ & -1.324920 & -4.963526 \\
\hline H & 1.834510 & $-\odot .0 \odot \odot 3 \odot \odot$ & -4.021853 \\
\hline C & -2.027347 & 1.321892 & $\odot .815483$ \\
\hline $\mathrm{H}$ & -2.223606 & 2.126599 & 1.532941 \\
\hline $\mathrm{H}$ & -1.195845 & 1.656041 & $\odot .196708$ \\
\hline $\mathrm{H}$ & -2.915279 & 1.225823 & $\odot .177593$ \\
\hline C & -2.858422 & $-\odot .0 \odot \odot 295$ & 2.696557 \\
\hline $\mathrm{H}$ & -2.820535 & ๑.889052 & 3.334298 \\
\hline $\mathrm{H}$ & -3.835926 & $-\odot .0 \odot \odot 298$ & 2.199755 \\
\hline H & -2.820487 & $-\odot .889748$ & 3.334157 \\
\hline C & -2.027078 & -1.322205 & $\odot .815433$ \\
\hline $\mathrm{H}$ & -2.223053 & -2.127018 & 1.532854 \\
\hline $\mathrm{H}$ & -2.915094 & -1.226356 & $\odot .177629$ \\
\hline $\mathrm{H}$ & -1 & 56060 & 0.196 \\
\hline
\end{tabular}


Compound 1e: ground state GS-1

B3LYP/6-31G(d) Energy: -1040.84198903 a.u. Imaginary frequencies: 0

$\begin{array}{lrrr}\text { C } & -0.355024 & 0.258722 & -2.546589 \\ \mathrm{C} & 0.200955 & 0.433668 & -3.814565 \\ \mathrm{C} & 1.543414 & 0.130504 & -4.032221 \\ \mathrm{C} & 2.325122 & -0.340936 & -2.977576 \\ \mathrm{C} & 1.755644 & -0.511509 & -1.718472 \\ \mathrm{C} & 0.402142 & -0.223358 & -1.463851 \\ \mathrm{C} & -1.241562 & -1.255145 & 0.153305 \\ \mathrm{C} & -0.133440 & -0.432187 & -0.089461 \\ \mathrm{C} & 0.515271 & 0.162805 & 1.003835 \\ \mathrm{C} & 0.082482 & -0.032277 & 2.319791 \\ \mathrm{C} & -1.029382 & -0.859273 & 2.532852 \\ \mathrm{C} & -1.681015 & -1.465541 & 1.459458 \\ \mathrm{H} & -0.419823 & 0.814939 & -4.618383 \\ \mathrm{H} & 1.971753 & 0.266871 & -5.021178 \\ \mathrm{H} & 3.372769 & -0.581402 & -3.134590 \\ \mathrm{H} & 2.357961 & -0.895230 & -0.900012 \\ \mathrm{H} & -1.749106 & -1.737477 & -0.675887 \\ \mathrm{H} & 1.369964 & 0.809601 & 0.817837 \\ \mathrm{H} & -2.537164 & -2.110093 & 1.641130 \\ \mathrm{C} & -0.108378 & 1.682780 & 4.175767 \\ \mathrm{C} & 0.798989 & 0.652127 & 3.476969 \\ \mathrm{H} & -0.483076 & 2.426898 & 3.464657 \\ \mathrm{H} & -0.975886 & 1.197753 & 4.639029 \\ \mathrm{H} & 0.440354 & 2.209820 & 4.965588 \\ \mathrm{C} & 1.364750 & -0.362009 & 4.488594 \\ \mathrm{H} & 1.650093 & 1.200269 & 3.050269 \\ \mathrm{H} & 2.032224 & -1.080297 & 4.000034 \\ \mathrm{H} & 1.931847 & 0.151830 & 5.273885 \\ \mathrm{H} & 0.563211 & -0.929409 & 4.976403 \\ \mathrm{H} & -1.391181 & -1.032034 & 3.543497 \\ \mathrm{Cl} & -2.045514 & 0.721043 & -2.342900\end{array}$


Compound 1e: ground state GS-2

B3LYP/6-31G(d) Energy: -1040.84204961 a.u. Imaginary frequencies: 0

$\begin{array}{lrrr}\mathrm{C} & 0.777472 & -0.091552 & 2.254699 \\ \mathrm{C} & 0.939069 & 0.225649 & 3.604036 \\ \mathrm{C} & -0.143475 & 0.703743 & 4.339589 \\ \mathrm{C} & -1.385092 & 0.855840 & 3.722433 \\ \mathrm{C} & -1.530079 & 0.536542 & 2.374985 \\ \mathrm{C} & -0.457543 & 0.059517 & 1.599010 \\ \mathrm{C} & -1.739030 & -1.119100 & -0.197553 \\ \mathrm{C} & -0.694311 & -0.256956 & 0.162639 \\ \mathrm{C} & 0.068807 & 0.337507 & -0.854184 \\ \mathrm{C} & -0.181915 & 0.088158 & -2.206781 \\ \mathrm{C} & -1.229606 & -0.784205 & -2.538366 \\ \mathrm{C} & -1.999655 & -1.381663 & -1.542648 \\ \mathrm{Cl} & 2.183278 & -0.746221 & 1.412604 \\ \mathrm{H} & 1.909024 & 0.086488 & 4.069558 \\ \mathrm{H} & -0.012808 & 0.951159 & 5.389264 \\ \mathrm{H} & -2.236691 & 1.227795 & 4.284979 \\ \mathrm{H} & -2.491430 & 0.671513 & 1.887622 \\ \mathrm{H} & -2.333615 & -1.595077 & 0.577363 \\ \mathrm{H} & 0.870153 & 1.019335 & -0.583222 \\ \mathrm{H} & -2.805943 & -2.058378 & -1.813710 \\ \mathrm{C} & 1.452995 & -0.279411 & -4.111018 \\ \mathrm{C} & 0.666709 & 0.754307 & -3.282632 \\ \mathrm{H} & 2.079450 & -0.907271 & -3.468459 \\ \mathrm{H} & 0.778226 & -0.939846 & -4.668676 \\ \mathrm{H} & 2.102385 & 0.222875 & -4.838013 \\ \mathrm{C} & -0.172933 & 1.669737 & -4.193441 \\ \mathrm{H} & 1.400591 & 1.387987 & -2.766703 \\ \mathrm{H} & -0.703743 & 2.430579 & -3.610814 \\ \mathrm{H} & 0.467620 & 2.181693 & -4.921393 \\ \mathrm{H} & -0.920839 & 1.096831 & -4.754760 \\ \mathrm{H} & -1.446577 & -0.998634 & -3.581913\end{array}$


Compound 1e: transition state TS-180

B3LYP/6-31G(d) Energy: -1040.83039195 a.u. Imaginary frequencies: 1

\begin{tabular}{|c|c|c|c|}
\hline C & $\odot .215261$ & $\odot .0 \odot \odot \odot 17$ & -2.677440 \\
\hline C & -0.523514 & -0.000016 & -3.865981 \\
\hline C & -1.910888 & $-\odot .00 \odot \odot 26$ & -3.849739 \\
\hline C & -2.557021 & -๑.๑०९०००९ & -2.616374 \\
\hline C & -1.812192 & $-\odot .00 \odot \odot \odot 6$ & -1.445667 \\
\hline C & $-\odot .395536$ & $\odot . ๑ \odot \odot \odot \odot \odot$ & -1.396637 \\
\hline C & 1.669286 & $-\odot .00 \odot \odot 2 \odot$ & 0.145975 \\
\hline C & $\odot .274799$ & $-\odot .000 \odot 42$ & -0.047031 \\
\hline C & -0.507413 & $-\odot .0 \odot \odot \odot 72$ & 1.131716 \\
\hline C & $\odot .032275$ & -๑.๑๑९९71 & 2.418795 \\
\hline C & 1.426040 & -๑.๑०९९०61 & 2.559401 \\
\hline C & 2.227190 & $-\odot .000032$ & 1.424585 \\
\hline $\mathrm{Cl}$ & 1.963519 & ๑. $00 \odot \odot 41$ & -2.979378 \\
\hline $\mathrm{H}$ & 0.015421 & $-\odot .00 \odot \odot 3 \odot$ & -4.806988 \\
\hline $\mathrm{H}$ & -2.468770 & $-\odot .00 \odot \odot 42$ & -4.781420 \\
\hline $\mathrm{H}$ & -3.641707 & $-\odot . \odot \odot \odot \odot \odot 7$ & -2.557778 \\
\hline $\mathrm{H}$ & -2.369322 & -0.000028 & -0.519873 \\
\hline $\mathrm{H}$ & 2.343974 & - ๑. ๑०९०14 & -0.692136 \\
\hline $\mathrm{H}$ & -1.588461 & -๑. ๑०९९८6 & 1.075009 \\
\hline $\mathrm{H}$ & 3.309649 & -0.000021 & 1.524003 \\
\hline C & -0.707064 & -1.269898 & 4.487954 \\
\hline C & -0.886169 & $-\odot .00 \odot \odot 14$ & 3.634567 \\
\hline $\mathrm{H}$ & -0.869823 & -2.174203 & 3.891509 \\
\hline $\mathrm{H}$ & $\odot .302851$ & -1.326911 & 4.911357 \\
\hline $\mathrm{H}$ & -1.417907 & -1.278234 & 5.322899 \\
\hline C & -0.707065 & 1.270084 & 4.487675 \\
\hline $\mathrm{H}$ & -1.919292 & - ๑. ๑०९९৪९ & 3.260607 \\
\hline $\mathrm{H}$ & $-\odot .867741$ & 2.174296 & 3.890530 \\
\hline $\mathrm{H}$ & -1.419324 & 1.279630 & 5.321398 \\
\hline $\mathrm{H}$ & 0.302198 & 1.326092 & 4.912775 \\
\hline & 0 & 00063 & 3.54 \\
\hline
\end{tabular}


Compound 1e: transition state TS- $\odot$

B3LYP/6-31G(d) Energy: -1040.83033182 a.u. Imaginary frequencies: 1

$\begin{array}{lrrr}\text { C } & -0.835602 & -0.000069 & 2.210361 \\ \mathrm{C} & -1.033190 & -0.000096 & 3.595946 \\ \mathrm{C} & 0.040303 & -0.000019 & 4.474849 \\ \mathrm{C} & 1.327874 & 0.000107 & 3.944729 \\ \mathrm{C} & 1.509216 & 0.000108 & 2.569198 \\ \mathrm{C} & 0.455160 & -0.000009 & 1.621227 \\ \mathrm{C} & 2.169421 & -0.000076 & -0.239212 \\ \mathrm{C} & 0.813839 & -0.000024 & 0.157439 \\ \mathrm{C} & -0.129999 & 0.000032 & -0.889973 \\ \mathrm{C} & 0.224307 & 0.000021 & -2.244347 \\ \mathrm{C} & 1.579419 & -0.000056 & -2.586912 \\ \mathrm{C} & 2.540519 & -0.000101 & -1.579715 \\ \mathrm{Cl} & -2.370260 & 0.000029 & 1.319655 \\ \mathrm{H} & -2.050843 & -0.000155 & 3.970612 \\ \mathrm{H} & -0.130694 & -0.000037 & 5.547231 \\ \mathrm{H} & 2.196732 & 0.000200 & 4.596709 \\ \mathrm{H} & 2.530848 & 0.000160 & 2.217441 \\ \mathrm{H} & 2.970877 & -0.000046 & 0.487123 \\ \mathrm{H} & -1.183256 & 0.000058 & -0.666100 \\ \mathrm{H} & 3.596878 & -0.000150 & -1.836066 \\ \mathrm{C} & -0.808972 & 1.269760 & -4.183564 \\ \mathrm{C} & -0.862571 & 0.000052 & -3.313195 \\ \mathrm{H} & -0.884477 & 2.174113 & -3.570078 \\ \mathrm{H} & 0.129090 & 1.326523 & -4.748713 \\ \mathrm{H} & -1.633354 & 1.277099 & -4.906564 \\ \mathrm{C} & -0.809130 & -1.269731 & -4.183461 \\ \mathrm{H} & -1.828857 & 0.000140 & -2.791444 \\ \mathrm{H} & -0.884098 & -2.174044 & -3.569850 \\ \mathrm{H} & -1.633888 & -1.277332 & -4.906028 \\ \mathrm{H} & 0.128652 & -1.326327 & -4.749098 \\ \mathrm{H} & 1.887248 & -0.000078 & -3.629518\end{array}$


Compound 1f: ground state GS-1

B3LYP/6-31G(d) Energy: -3152.35204342 a.u. Imaginary frequencies: 0

$\begin{array}{lrrr}\mathrm{C} & 0.193324 & -0.104833 & 2.247266 \\ \mathrm{C} & 0.877039 & -0.307823 & 3.445970 \\ \mathrm{C} & 2.262448 & -0.163007 & 3.487879 \\ \mathrm{C} & 2.955684 & 0.180535 & 2.327319 \\ \mathrm{C} & 2.257732 & 0.382569 & 1.139567 \\ \mathrm{C} & 0.858525 & 0.250966 & 1.061487 \\ \mathrm{C} & -0.848588 & 1.421952 & -0.385394 \\ \mathrm{C} & 0.188371 & 0.488807 & -0.247505 \\ \mathrm{C} & 0.639961 & -0.189233 & -1.390426 \\ \mathrm{C} & 0.080490 & 0.030651 & -2.653735 \\ \mathrm{C} & -0.955506 & 0.968971 & -2.761812 \\ \mathrm{C} & -1.412285 & 1.657082 & -1.638268 \\ \mathrm{H} & 0.321986 & -0.586764 & 4.335001 \\ \mathrm{H} & 2.791474 & -0.321218 & 4.423429 \\ \mathrm{H} & 4.035451 & 0.298244 & 2.346651 \\ \mathrm{H} & 2.794479 & 0.669018 & 0.239701 \\ \mathrm{H} & -1.206775 & 1.963304 & 0.483921 \\ \mathrm{H} & 1.438142 & -0.920789 & -1.284911 \\ \mathrm{H} & -2.211490 & 2.386791 & -1.739499 \\ \mathrm{C} & -0.501684 & -1.652456 & -4.460486 \\ \mathrm{C} & 0.587428 & -0.738519 & -3.867055 \\ \mathrm{H} & -0.889780 & -2.346798 & -3.707478 \\ \mathrm{H} & -1.346963 & -1.067736 & -4.842565 \\ \mathrm{H} & -0.099868 & -2.240568 & -5.294231 \\ \mathrm{C} & 1.167668 & 0.199020 & -4.942566 \\ \mathrm{H} & 1.404819 & -1.385465 & -3.520129 \\ \mathrm{H} & 1.967022 & 0.826389 & -4.533287 \\ \mathrm{H} & 1.580725 & -0.380597 & -5.776748 \\ \mathrm{H} & 0.396993 & 0.863874 & -5.350476 \\ \mathrm{H} & -1.411015 & 1.165686 & -3.729230 \\ \mathrm{Br} & -1.704824 & -0.384899 & 2.279326 \\ & & & \end{array}$


Compound 1f: ground state GS-2

B3LYP/6-31G(d) Energy: -3152.35211234 a.u. Imaginary frequencies: 0

$\begin{array}{lrrr}\mathrm{C} & -0.298527 & 0.103298 & -2.115629 \\ \mathrm{C} & -0.321508 & 0.447491 & -3.467036 \\ \mathrm{C} & 0.864818 & 0.778424 & -4.119140 \\ \mathrm{C} & 2.068331 & 0.758363 & -3.414464 \\ \mathrm{C} & 2.073446 & 0.415562 & -2.064934 \\ \mathrm{C} & 0.894130 & 0.082139 & -1.372728 \\ \mathrm{C} & 1.887110 & -1.260675 & 0.489478 \\ \mathrm{C} & 0.986471 & -0.271710 & 0.071210 \\ \mathrm{C} & 0.231696 & 0.405375 & 1.041787 \\ \mathrm{C} & 0.348375 & 0.112362 & 2.403599 \\ \mathrm{C} & 1.250402 & -0.889335 & 2.793224 \\ \mathrm{C} & 2.012453 & -1.567847 & 1.844457 \\ \mathrm{Br} & -1.974776 & -0.396031 & -1.325090 \\ \mathrm{H} & -1.265145 & 0.444344 & -4.001380 \\ \mathrm{H} & 0.842962 & 1.046540 & -5.171663 \\ \mathrm{H} & 2.999981 & 1.015235 & -3.910605 \\ \mathrm{H} & 3.007538 & 0.417573 & -1.510448 \\ \mathrm{H} & 2.473645 & -1.799920 & -0.249348 \\ \mathrm{H} & -0.460857 & 1.180418 & 0.725852 \\ \mathrm{H} & 2.705669 & -2.343457 & 2.159739 \\ \mathrm{C} & -1.517710 & -0.070214 & 4.105619 \\ \mathrm{C} & -0.496338 & 0.862092 & 3.426015 \\ \mathrm{H} & -2.158510 & -0.560445 & 3.364859 \\ \mathrm{H} & -1.015999 & -0.854750 & 4.684804 \\ \mathrm{H} & -2.158716 & 0.494005 & 4.793755 \\ \mathrm{C} & 0.366565 & 1.594006 & 4.470377 \\ \mathrm{H} & -1.064358 & 1.624671 & 2.876158 \\ \mathrm{H} & 1.072987 & 2.280372 & 3.990888 \\ \mathrm{H} & -0.265267 & 2.174987 & 5.152616 \\ \mathrm{H} & 0.945870 & 0.887640 & 5.076903 \\ \mathrm{H} & 1.359062 & -1.142127 & 3.844993\end{array}$


Compound 1f: transition state TS-180

B3LYP/6-31G(d) Energy: -3152.33978731 a.u. Imaginary frequencies: 1

$\begin{array}{lrrr}\mathrm{C} & 0.358530 & -0.000018 & 2.253539 \\ \mathrm{C} & 1.248237 & -0.000023 & 3.334257 \\ \mathrm{C} & 2.621789 & -0.000034 & 3.136920 \\ \mathrm{C} & 3.099795 & -0.000020 & 1.829705 \\ \mathrm{C} & 2.206825 & -0.000017 & 0.768044 \\ \mathrm{C} & 0.795142 & -0.000033 & 0.903643 \\ \mathrm{C} & -1.443250 & -0.000351 & -0.377858 \\ \mathrm{C} & -0.036208 & -0.000078 & -0.355100 \\ \mathrm{C} & 0.595251 & 0.000094 & -1.621431 \\ \mathrm{C} & -0.097690 & 0.000044 & -2.832690 \\ \mathrm{C} & -1.498222 & -0.000209 & -2.802776 \\ \mathrm{C} & -2.153936 & -0.000410 & -1.578354 \\ \mathrm{Br} & -1.475424 & 0.000115 & 2.869321 \\ \mathrm{H} & 0.840975 & -0.000010 & 4.338879 \\ \mathrm{H} & 3.296092 & -0.000043 & 3.988173 \\ \mathrm{H} & 4.167224 & -0.000005 & 1.628143 \\ \mathrm{H} & 2.638262 & 0.000011 & -0.222261 \\ \mathrm{H} & -2.012173 & -0.000528 & 0.535511 \\ \mathrm{H} & 1.674681 & 0.000268 & -1.700050 \\ \mathrm{H} & -3.240420 & -0.000619 & -1.543638 \\ \mathrm{C} & 0.382273 & -1.269767 & -4.976240 \\ \mathrm{C} & 0.665526 & 0.000220 & -4.151983 \\ \mathrm{H} & 0.620460 & -2.173962 & -4.405559 \\ \mathrm{H} & -0.673169 & -1.327947 & -5.267745 \\ \mathrm{H} & 0.981449 & -1.276767 & -5.894619 \\ \mathrm{C} & 0.381537 & 1.269969 & -4.976354 \\ \mathrm{H} & 1.736686 & 0.000542 & -3.907135 \\ \mathrm{H} & 0.619054 & 2.174356 & -4.405701 \\ \mathrm{H} & 0.980830 & 1.277305 & -5.894654 \\ \mathrm{H} & -0.673903 & 1.327437 & -5.268010 \\ \mathrm{H} & -2.069777 & -0.000263 & -3.727496\end{array}$


Compound 1f: transition state TS- $\odot$

B3LYP/6-31G(d) Energy: -3152.33973461 a.u. Imaginary frequencies: 1

$\begin{array}{lrrr}\mathrm{C} & -0.313949 & -0.000061 & -2.091014 \\ \mathrm{C} & -0.379140 & -0.000193 & -3.489358 \\ \mathrm{C} & 0.771329 & 0.000173 & -4.265028 \\ \mathrm{C} & 2.003042 & 0.000760 & -3.616679 \\ \mathrm{C} & 2.053113 & 0.000784 & -2.230446 \\ \mathrm{C} & 0.915530 & 0.000222 & -1.383444 \\ \mathrm{C} & 2.466505 & -0.000656 & 0.617236 \\ \mathrm{C} & 1.149317 & -0.000136 & 0.106760 \\ \mathrm{C} & 0.122269 & 0.000086 & 1.071861 \\ \mathrm{C} & 0.361306 & -0.000078 & 2.451337 \\ \mathrm{C} & 1.682376 & -0.000484 & 2.907373 \\ \mathrm{C} & 2.724186 & -0.000807 & 1.984079 \\ \mathrm{Br} & -2.088546 & -0.000337 & -1.319285 \\ \mathrm{H} & -1.355203 & -0.000537 & -3.960656 \\ \mathrm{H} & 0.699686 & 0.000069 & -5.348611 \\ \mathrm{H} & 2.929779 & 0.001203 & -4.183401 \\ \mathrm{H} & 3.037687 & 0.001384 & -1.785773 \\ \mathrm{H} & 3.327213 & -0.001114 & -0.037423 \\ \mathrm{H} & -0.909337 & 0.000422 & 0.762278 \\ \mathrm{H} & 3.755572 & -0.001209 & 2.327354 \\ \mathrm{C} & -0.832094 & -1.268992 & 4.296917 \\ \mathrm{C} & -0.812210 & 0.000236 & 3.424341 \\ \mathrm{H} & -0.855200 & -2.173739 & 3.679814 \\ \mathrm{H} & 0.054879 & -1.325045 & 4.939397 \\ \mathrm{H} & -1.714520 & -1.276242 & 4.947860 \\ \mathrm{C} & -0.832415 & 1.270387 & 4.295561 \\ \mathrm{H} & -1.730977 & -0.000193 & 2.823034 \\ \mathrm{H} & -0.856868 & 2.174439 & 3.677489 \\ \mathrm{H} & -1.714249 & 1.277594 & 4.947311 \\ \mathrm{H} & 0.055121 & 1.327900 & 4.937129 \\ \mathrm{H} & 1.900548 & -0.000582 & 3.972219\end{array}$


Compound 1h: ground state GS-anti

B3LYP/6-31G(d) Energy: -930.24641166 a.u. Imaginary frequencies: $\odot$

\begin{tabular}{lrrr} 
C & 1.237657 & 0.616440 & 3.495011 \\
$\mathrm{C}$ & 0.637577 & 0.308605 & 2.262587 \\
$\mathrm{C}$ & -0.637825 & -0.308770 & 2.262447 \\
$\mathrm{C}$ & -1.238163 & -0.616636 & 3.494736 \\
$\mathrm{C}$ & -0.622490 & -0.314739 & 4.707229 \\
$\mathrm{C}$ & 0.621731 & 0.314511 & 4.707367 \\
$\mathrm{H}$ & 2.222971 & 1.074684 & 3.491258 \\
$\mathrm{H}$ & -2.223477 & -1.074878 & 3.490765 \\
$\mathrm{C}$ & -1.403438 & -0.610243 & 1.017357 \\
$\mathrm{C}$ & -1.662757 & 0.388995 & 0.065189 \\
$\mathrm{C}$ & -2.430055 & 0.143655 & -1.077101 \\
$\mathrm{C}$ & -2.943232 & -1.148652 & -1.268029 \\
$\mathrm{C}$ & -2.698019 & -2.154159 & -0.335008 \\
$\mathrm{C}$ & -1.938052 & -1.888901 & 0.804927 \\
$\mathrm{H}$ & -3.540672 & -1.370985 & -2.148820 \\
$\mathrm{H}$ & -3.100197 & -3.151416 & -0.495317 \\
$\mathrm{H}$ & -1.744050 & -2.677572 & 1.527146 \\
$\mathrm{C}$ & 1.403450 & 0.610131 & 1.017667 \\
$\mathrm{C}$ & 1.938110 & 1.888799 & 0.805406 \\
$\mathrm{C}$ & 2.698328 & 2.154104 & -0.334350 \\
$\mathrm{C}$ & 2.943743 & 1.148636 & -1.267360 \\
$\mathrm{C}$ & 2.430505 & -0.143671 & -1.076611 \\
$\mathrm{C}$ & 1.662966 & -0.389064 & 0.065507 \\
$\mathrm{H}$ & 1.743952 & 2.677436 & 1.527619 \\
$\mathrm{H}$ & 3.100554 & 3.151363 & -0.494524 \\
$\mathrm{H}$ & 3.541374 & 1.371008 & -2.148011 \\
$\mathrm{H}$ & -1.117294 & -0.560042 & 5.643206 \\
$\mathrm{H}$ & 1.116338 & 0.559789 & 5.643455 \\
$\mathrm{H}$ & 1.260911 & -1.385653 & 0.227564 \\
$\mathrm{H}$ & -1.260730 & 1.385574 & 0.227377 \\
$\mathrm{C}$ & -2.701051 & 1.261276 & -2.076838 \\
$\mathrm{H}$ & -2.222300 & 2.167930 & -1.682929 \\
$\mathrm{C}$ & -4.206128 & 1.558382 & -2.214326 \\
$\mathrm{H}$ & -4.371977 & 2.405459 & -2.890727 \\
$\mathrm{H}$ & -4.748304 & 0.696923 & -2.622275 \\
$\mathrm{H}$ & -4.651488 & 1.803572 & -1.243979 \\
$\mathrm{C}$ & -2.067133 & 0.967306 & -3.449560 \\
$\mathrm{H}$ & -2.501956 & 0.069736 & -3.905627 \\
$\mathrm{H}$ & -2.231860 & 1.803975 & -4.139228 \\
$\mathrm{H}$ & -0.987055 & 0.807993 & -3.359025 \\
$\mathrm{C}$ & 2.701516 & -1.261192 & -2.076452 \\
$\mathrm{H}$ & 2.223651 & -2.168132 & -1.682123 \\
$\mathrm{C}$ & 2.066275 & -0.967628 & -3.448669 \\
$\mathrm{H}$ & 2.500136 & -0.069722 & -3.904995 \\
$\mathrm{H}$ & 2.231016 & -1.804143 & -4.138522 \\
$\mathrm{H}$ & 0.986165 & -0.809051 & -3.357248 \\
$\mathrm{C}$ & 4.206640 & -1.557416 & -2.215197 \\
$\mathrm{H}$ & 4.372362 & -2.404604 & -2.891488 \\
$\mathrm{H}$ & -0.695795 & -2.623932 \\
& & -1.802054 & -1.245186 \\
\hline
\end{tabular}


Compound 1h: ground state GS-syn

B3LYP/6-31G(d) Energy: -930.24638026 a.u. Imaginary frequencies: $\odot$

\begin{tabular}{|c|c|c|c|}
\hline C & 3.320455 & -0.398809 & -0.867878 \\
\hline C & 2.129527 & -0.041469 & -0.213358 \\
\hline C & 2.079098 & -0.129039 & 1.200781 \\
\hline C & 3.229608 & $-\odot .545091$ & 1.891683 \\
\hline C & 4.404240 & $-\odot .886387$ & 1.225585 \\
\hline C & 4.447929 & $-\odot .818935$ & -0.166242 \\
\hline $\mathrm{H}$ & 3.362601 & $-\odot .312191$ & -1.950160 \\
\hline $\mathrm{H}$ & 3.181630 & $-\odot .628474$ & 2.974009 \\
\hline C & $\odot .853035$ & 0.163770 & 1.999507 \\
\hline C & -0.375394 & -0.447553 & 1.700921 \\
\hline C & -1.524156 & $-\odot .214973$ & 2.462184 \\
\hline C & -1.430742 & $\odot .656618$ & 3.558074 \\
\hline C & $-\odot .219574$ & 1. 267511 & 3.876385 \\
\hline C & $\odot .917954$ & 1. 022891 & 3.106022 \\
\hline $\mathrm{H}$ & -2.308663 & $\odot .86 \odot 232$ & 4.166124 \\
\hline $\mathrm{H}$ & -0.160152 & 1.942145 & 4.726851 \\
\hline $\mathrm{H}$ & 1.857148 & 1.512124 & 3.350367 \\
\hline C & $\odot .994078$ & $\odot .450115$ & -1.048070 \\
\hline C & $\odot .357857$ & 1.670529 & -0.777786 \\
\hline C & -0.668335 & 2.125243 & -1.604282 \\
\hline C & -1.075968 & 1.374956 & -2.707323 \\
\hline C & $-\odot .456629$ & $\odot .153376$ & -3.006395 \\
\hline C & $\odot .574003$ & -0.286352 & -2.167962 \\
\hline $\mathrm{H}$ & $\odot .672310$ & 2.264040 & $\odot .074850$ \\
\hline $\mathrm{H}$ & $-1.15 \odot 267$ & 3.075735 & -1.389444 \\
\hline $\mathrm{H}$ & -1.879805 & 1.746373 & -3.338295 \\
\hline H & 5.274017 & -1.211164 & 1.790441 \\
\hline $\mathrm{H}$ & 5.355327 & -1.081151 & $-\odot .703758$ \\
\hline H & 1.056456 & -1.238261 & -2.380477 \\
\hline $\mathrm{H}$ & -0.432934 & -1.123695 & 0.852163 \\
\hline C & -2.841567 & -0.883266 & 2. 089301 \\
\hline $\mathrm{H}$ & -2.632912 & -1.558323 & 1.248403 \\
\hline C & -3.875758 & $\odot .15 \odot 272$ & 1.603045 \\
\hline $\mathrm{H}$ & -4.800220 & -0.346335 & 1.284159 \\
\hline $\mathrm{H}$ & -4.134171 & $\odot .857045$ & 2.400838 \\
\hline $\mathrm{H}$ & -3.486595 & $\odot .727233$ & $\odot .757184$ \\
\hline C & -3.408659 & -1.739100 & 3.236965 \\
\hline $\mathrm{H}$ & -3.658438 & -1.124810 & 4.110295 \\
\hline $\mathrm{H}$ & -4.325213 & -2.250584 & 2.919522 \\
\hline $\mathrm{H}$ & -2.687725 & -2.498594 & 3.558828 \\
\hline C & $-\odot .887044$ & $-\odot .684631$ & -4.203870 \\
\hline $\mathrm{H}$ & -0.243252 & -1.574774 & -4.221913 \\
\hline C & -2.341760 & -1.173000 & -4.068975 \\
\hline $\mathrm{H}$ & -3.045035 & $-\odot .331779$ & -4.058127 \\
\hline $\mathrm{H}$ & -2.611822 & -1.821835 & -4.910845 \\
\hline $\mathrm{H}$ & -2.484794 & -1.739086 & -3.142048 \\
\hline C & -0.676116 & $\odot .062891$ & -5.533948 \\
\hline $\mathrm{H}$ & $-\odot .932304$ & -0.580780 & -6.384023 \\
\hline $\mathrm{H}$ & -1.307275 & 0.957595 & -5.592781 \\
\hline $\mathrm{H}$ & 0.365578 & ๑. 381910 & -5.648342 \\
\hline
\end{tabular}


Compound 1h: transition state TS-180

B3LYP/6-31G(d) Energy: -930.23461274 a.u. Imaginary frequencies: 1

\begin{tabular}{|c|c|c|c|}
\hline C & 3.146513 & -0.104943 & -1.281529 \\
\hline C & 1.874011 & $\odot .097534$ & -0.719849 \\
\hline C & 1.678294 & -0.101086 & 0.678383 \\
\hline C & 2.819516 & $-\odot .496348$ & 1.412032 \\
\hline C & 4.071002 & $-\odot .691188$ & $\odot .840550$ \\
\hline C & 4.245801 & $-\odot .495384$ & $-\odot .526146$ \\
\hline $\mathrm{H}$ & 3.258621 & $\odot .055621$ & -2.350294 \\
\hline $\mathrm{H}$ & 2.741550 & $-\odot .663119$ & 2.477893 \\
\hline C & 0.374708 & $\odot .073421$ & 1.415114 \\
\hline C & $\odot .299208$ & -0.154277 & 2.808059 \\
\hline C & $-\odot .874010$ & $-\odot .011911$ & 3.551809 \\
\hline C & -2.044135 & 0.378066 & 2.887579 \\
\hline C & -2.008233 & $\odot .610555$ & 1.517633 \\
\hline C & $-\odot .826224$ & $\odot .462365$ & 0.792109 \\
\hline $\mathrm{H}$ & -2.975096 & $\odot .498465$ & 3.435854 \\
\hline $\mathrm{H}$ & -2.911948 & $\odot .913715$ & $\odot .994706$ \\
\hline $\mathrm{H}$ & -0.860917 & $\odot .655943$ & -0.266944 \\
\hline C & $\odot .839463$ & $\odot .519992$ & -1.722611 \\
\hline C & $\odot .649879$ & 1.874041 & -2.027314 \\
\hline C & -0.261738 & 2.247480 & -3.016013 \\
\hline C & -0.981113 & 1.279253 & -3.715116 \\
\hline C & $-\odot .79604 \odot$ & $-\odot .084707$ & -3.444002 \\
\hline C & 0.121367 & -0.441639 & -2.448885 \\
\hline $\mathrm{H}$ & 1.208789 & 2.629798 & -1.482332 \\
\hline $\mathrm{H}$ & $-\odot .408917$ & 3.300159 & -3.244242 \\
\hline $\mathrm{H}$ & -1.687436 & 1.589004 & -4.481449 \\
\hline $\mathrm{H}$ & 4.903850 & $-\odot .995885$ & 1.468872 \\
\hline $\mathrm{H}$ & 5.214285 & -0.641660 & $-\odot .996339$ \\
\hline H & $\odot .278274$ & -1.493989 & -2.222337 \\
\hline $\mathrm{H}$ & 1.179300 & -0.457353 & 3.362033 \\
\hline C & -0.868071 & -0.279123 & 5.052187 \\
\hline $\mathrm{H}$ & $\odot .157433$ & $-\odot .561707$ & 5.327421 \\
\hline C & -1.785900 & -1.458163 & 5.426944 \\
\hline $\mathrm{H}$ & -1.723294 & -1.671578 & 6.500845 \\
\hline $\mathrm{H}$ & -2.834000 & -1.236612 & 5.192641 \\
\hline $\mathrm{H}$ & -1.507171 & -2.366010 & 4.880887 \\
\hline C & -1.230469 & $\odot .981192$ & 5.860380 \\
\hline $\mathrm{H}$ & -2.253887 & 1.311320 & 5.645594 \\
\hline $\mathrm{H}$ & -1.164273 & $\odot .783128$ & 6.936981 \\
\hline $\mathrm{H}$ & $-\odot .555969$ & 1.811137 & 5.623144 \\
\hline C & -1.566643 & -1.157443 & -4.203375 \\
\hline $\mathrm{H}$ & -1.222919 & -2.129520 & -3.824561 \\
\hline C & -3.081097 & -1.068719 & -3.935013 \\
\hline $\mathrm{H}$ & -3.495209 & $-\odot .120918$ & -4.299384 \\
\hline $\mathrm{H}$ & -3.612289 & -1.881769 & -4.444199 \\
\hline $\mathrm{H}$ & -3.297906 & -1.137788 & -2.863467 \\
\hline C & -1.269583 & -1.120954 & -5.714209 \\
\hline $\mathrm{H}$ & -1.785222 & -1.940732 & -6.228472 \\
\hline $\mathrm{H}$ & -1.608710 & -0.180885 & -6.165503 \\
\hline $\mathrm{H}$ & -0.195991 & -1.216847 & -5.909658 \\
\hline
\end{tabular}


Compound 5: ground state GS

B3LYP/6-31G(d) Energy: -659.88051200 a.u. Imaginary frequencies: 0

\begin{tabular}{|c|c|c|c|}
\hline C & -0.416977 & ๑. 946832 & 2.352932 \\
\hline C & -0.907908 & ๑.819426 & 3.658137 \\
\hline C & -1.140285 & -0.430689 & 4.232699 \\
\hline C & -0.883148 & -1.586469 & 3.497632 \\
\hline C & -0.393394 & -1.476854 & 2.195569 \\
\hline C & -0.153907 & $-\odot .225824$ & 1.611358 \\
\hline C & 1.742054 & -0.131975 & -0.071742 \\
\hline C & $\odot .359679$ & $-\odot .157516$ & 0.204321 \\
\hline C & -0.555907 & $-\odot .133924$ & -0.856418 \\
\hline C & -0.152689 & -0.080825 & -2.195264 \\
\hline C & 1.222849 & $-\odot .052075$ & -2.455386 \\
\hline C & 2.145567 & $-\odot .078607$ & -1.411591 \\
\hline C & -0.189138 & 2.317895 & 1.756995 \\
\hline $\mathrm{H}$ & -1.114222 & 1.720001 & 4.232512 \\
\hline $\mathrm{H}$ & -1.522224 & $-\odot .49820 \odot$ & 5.248095 \\
\hline $\mathrm{H}$ & -1.062768 & -2.567005 & 3.930621 \\
\hline $\mathrm{H}$ & -0.193406 & -2.373283 & 1.613751 \\
\hline $\mathrm{H}$ & -1.618692 & -0.161210 & -0.623465 \\
\hline $\mathrm{H}$ & 3.209084 & $-\odot .059878$ & -1.640486 \\
\hline $\mathrm{H}$ & $-\odot .80700 \odot$ & 2.476786 & 0.865321 \\
\hline $\mathrm{H}$ & ๑.852025 & 2.457539 & 1.442291 \\
\hline $\mathrm{H}$ & -0.430613 & 3.103609 & 2.479738 \\
\hline C & -1.104180 & 1.240765 & -4.141900 \\
\hline C & -1.182590 & $-\odot .057874$ & -3.317024 \\
\hline $\mathrm{H}$ & -1.220852 & 2.123311 & -3.503465 \\
\hline $\mathrm{H}$ & -0.140180 & 1.327145 & -4.657409 \\
\hline $\mathrm{H}$ & -1.892119 & 1.263531 & -4.904340 \\
\hline C & -1.069911 & -1.296343 & -4.226156 \\
\hline $\mathrm{H}$ & -2.173938 & $-\odot .087235$ & -2.844314 \\
\hline $\mathrm{H}$ & -1.162443 & -2.221933 & -3.647592 \\
\hline $\mathrm{H}$ & -1.857866 & -1.289436 & -4.988881 \\
\hline $\mathrm{H}$ & -0.104530 & -1.322596 & -4.745665 \\
\hline $\mathrm{H}$ & 1.583504 & $-\odot .010644$ & -3.480478 \\
\hline C & 2.769448 & $-\odot .171407$ & 1.036721 \\
\hline $\mathrm{H}$ & 2.652302 & 0.668520 & 1.732340 \\
\hline $\mathrm{H}$ & 2.681745 & -1.085235 & 1.636256 \\
\hline $\mathrm{H}$ & 3.784826 & -0.130999 & 0.629870 \\
\hline
\end{tabular}


Compound 5: transition state TS-180

B3LYP/6-31G(d) Energy: -659.85394200 a.u. Imaginary frequencies: 1

\begin{tabular}{|c|c|c|c|}
\hline C & -1.404249 & $-\odot .0 \odot \odot 254$ & 2.041847 \\
\hline C & -1.753290 & $-\odot .000591$ & 3.402780 \\
\hline C & -0.829293 & -0.000438 & 4.440283 \\
\hline C & 0.515862 & $\odot .00 \odot 296$ & 4.105258 \\
\hline C & $\odot .890049$ & $\odot .000585$ & 2.765521 \\
\hline C & -0.010497 & $\odot .000 \odot 93$ & 1.676096 \\
\hline C & 1.936960 & $-0.000 \odot 23$ & -0.096938 \\
\hline C & 0.543765 & $-\odot .0 \odot \odot \odot 48$ & 0.261613 \\
\hline C & $-\odot .354248$ & $-\odot .000189$ & $-\odot .830270$ \\
\hline C & -0.002127 & -0.000277 & -2.180886 \\
\hline C & 1.354764 & -0.000259 & -2.493370 \\
\hline C & 2.279244 & -0.000119 & -1.458925 \\
\hline C & -2.617762 & -0.000055 & 1.124513 \\
\hline $\mathrm{H}$ & -2.811201 & $-\odot .00 \odot 882$ & 3.652063 \\
\hline $\mathrm{H}$ & -1.159874 & $-\odot .000757$ & 5.475287 \\
\hline $\mathrm{H}$ & 1.285967 & 0.000633 & 4.872255 \\
\hline $\mathrm{H}$ & 1.944564 & $\odot .001232$ & 2.581928 \\
\hline $\mathrm{H}$ & -1.408269 & -0.000303 & -0.639840 \\
\hline $\mathrm{H}$ & 3.335908 & $-\odot .0 \odot \odot \odot 97$ & -1.714075 \\
\hline $\mathrm{H}$ & -2.667667 & -0.886247 & $\odot .481768$ \\
\hline $\mathrm{H}$ & -2.667122 & $\odot .885969$ & 0.481520 \\
\hline $\mathrm{H}$ & -3.528425 & $\odot .00 \odot 286$ & 1.730260 \\
\hline C & -1.015998 & 1.269636 & -4.128614 \\
\hline C & -1.078078 & -0.000278 & -3.258998 \\
\hline $\mathrm{H}$ & -1.097846 & 2.173882 & -3.515666 \\
\hline $\mathrm{H}$ & -0.071086 & 1.326385 & -4.682161 \\
\hline $\mathrm{H}$ & -1.831679 & 1.277645 & -4.861591 \\
\hline C & -1.014376 & -1.268767 & -4.130553 \\
\hline $\mathrm{H}$ & -2.051152 & -0.001306 & -2.748649 \\
\hline $\mathrm{H}$ & -1.094310 & -2.174079 & -3.518928 \\
\hline $\mathrm{H}$ & -1.830523 & -1.277027 & -4.863002 \\
\hline $\mathrm{H}$ & $-\odot .069771$ & -1.323112 & -4.684862 \\
\hline $\mathrm{H}$ & 1.698376 & $-\odot .00 \odot 401$ & -3.524810 \\
\hline C & 3.151752 & $\odot .000233$ & 0.818312 \\
\hline $\mathrm{H}$ & 3.204418 & ๑.887035 & 1.460377 \\
\hline & 3.203826 & -0.885513 & 1.461839 \\
\hline & 4.0609 & 0.00061 & \\
\hline
\end{tabular}

